\title{
EVIDENCE FOR PERIODICITY IN 43 YEAR-LONG MONITORING OF NGC 5548
}

\author{
E. Bon ${ }^{1,2}$, S. Zucker ${ }^{3}$, H. Netzer ${ }^{4}$, P. Marziani ${ }^{5}$, N. Bon ${ }^{1,2}$, P. Jovanović ${ }^{1,2}$, A. I. Shapovalova ${ }^{6}$, S. Komossa $^{7}$, \\ C. M. Gaskell ${ }^{8}$, L. Č. Popović ${ }^{1,2}$, S. Britzen ${ }^{7}$, V. H. Chavushyan ${ }^{9}$, A. N. Burenkov ${ }^{6}$, S. Sergeev ${ }^{10}$, G. La Mura ${ }^{11}$, \\ J. R. VAldÉs ${ }^{9}$, AND M. STALEVSKi ${ }^{1,12,13}$ \\ ${ }^{1}$ Astronomical Observatory, Volgina 7, 11060 Belgrade, Serbia \\ ${ }^{2}$ Isaac Newton Institute of Chile, Yugoslavia Branch Belgrade, Serbia \\ ${ }^{3}$ Department of Geosciences, Tel-Aviv University, Tel-Aviv 6997801, Israel \\ ${ }^{4}$ School of Physics and Astronomy and the Wise Observatory, The Raymond and Beverly Sackler Faculty of Exact Sciences, \\ Tel-Aviv University, Tel-Aviv 6997801, Israel \\ 5 INAF, Osservatorio Astronomico di Padova, Padova, Italy \\ ${ }^{6}$ Special Astrophysical Observatory of the Russian AS, Nizhnij Arkhyz, Karachaevo-Cherkesia 369167, Russia \\ Max-Planck-Institut für Radioastronomie, Auf dem Hügel 69, 53121 Bonn, Germany \\ ${ }^{8}$ Department of Astronomy and Astrophysics, University of California at Santa Cruz, Santa Cruz, CA 95064, USA \\ ${ }^{9}$ Instituto Nacional de Astrofsica, Óptica y Electrónica, Apartado Postal 51, CP 72000, Puebla, Pue, Mexico, Mexico \\ ${ }^{10}$ Crimean Astrophysical Observatory, P/O Nauchny, Republic of Crimea 298409, Russia \\ ${ }^{11}$ Dipartimento di Fisica e Astronomia "G. Galilei," Università degli Studi di Padova, Vicolo dell'Osservatorio 3, I-35122—Padova, Italy \\ ${ }^{12}$ Departamento de Astronomía, Universidad de Chile, Camino El Observatorio 1515, Casilla 36-D Santiago, Chile \\ ${ }^{13}$ Sterrenkundig Observatorium, Universiteit Gent, Krijgslaan 281-S9, Gent, B-9000, Belgium \\ Received 2016 April 10; revised 2016 June 11; accepted 2016 June 14; published 2016 August 23
}

\begin{abstract}
We present an analysis of 43 years (1972 to 2015) of spectroscopic observations of the Seyfert 1 galaxy NGC 5548. This includes 12 years of new unpublished observations (2003 to 2015). We compiled about $1600 \mathrm{H} \beta$ spectra and analyzed the long-term spectral variations of the $5100 \AA$ continuum and the $\mathrm{H} \beta$ line. Our analysis is based on standard procedures, including the Lomb-Scargle method, which is known to be rather limited to such heterogeneous data sets, and a new method developed specifically for this project that is more robust and reveals a $\sim 5700$ day periodicity in the continuum light curve, the $\mathrm{H} \beta$ light curve, and the radial velocity curve of the red wing of the $\mathrm{H} \beta$ line. The data are consistent with orbital motion inside the broad emission line region of the source. We discuss several possible mechanisms that can explain this periodicity, including orbiting dusty and dust-free clouds, a binary black hole system, tidal disruption events, and the effect of an orbiting star periodically passing through an accretion disk.
\end{abstract}

Key words: accretion, accretion disks - galaxies: active - galaxies: nuclei - galaxies: Seyfert - quasars: individual (NGC 5548) - quasars: supermassive black holes

Supporting material: machine-readable tables

\section{INTRODUCTION}

Despite much progress in recent years, many fundamental questions about the structure and kinematics of the innermost material in active galactic nuclei (AGNs) remain unanswered. Two key features of thermal AGNs are (i) that the IR to X-ray continuum is highly variable and (ii) that they have a broadline region (BLR). Because the wavelengths of emission lines are well known, the effective line of sight velocity of line-emitting gas is known. The line profiles of broad lines are thus an important constraint on models of the inner regions of AGNs. Furthermore, the ratios of intensities of different lines depend on the physical conditions of the environment of the gas, such as the density and radiation field.

The variability of AGNs on short and long timescales may provide valuable insights about the physics of accretion, mechanisms of fueling nuclei, and the growth of supermassive black holes (BHs). Because the BLR gas is close to the center of the AGN, it readily responds to continuum variability. Cross correlating the variability of broad lines with the variability of the continuum gives the sizes of line-emitting regions (Cherepashchuk \& Lyutyi 1973; Gaskell \& Sparke 1986). In addition, the velocity dependence of a line's response to the continuum variability provides information about the kinematics and dynamics of the line-emitting gas (see, e.g.,
Gaskell 1988; Maoz et al. 1994; Netzer \& Peterson 1997; Peterson 1997; Netzer 2013).

Because of its brightness, NGC 5548 was among the first Seyfert galaxies to be studied. It was the first galaxy to have optical variability reported (Deutsch 1966). Between 1966 (Dibai et al. 1968) and 1970-71 (Anderson 1971; Ulrich 1972) there was a large change in the profile of the broad $\mathrm{H} \alpha$ line, and the optical continuum was highly variable (Lyutyi 1973). The first reverberation mapping of NGC 5548 (Peterson \& Gaskell 1986) showed that $\mathrm{H} \beta$ responded to continuum changes with a delay of only a few weeks. Because its brightness, reliable variability, and convenient BLR size, NGC 5548 was seen as an easy target for reverberation mapping, and has been the subject of much monitoring for several decades (see e.g., Peterson \& Gaskell 1986; Netzer et al. 1990; Clavel et al. 1991; Koratkar \& Gaskell 1991; Peterson et al. 1991, 1992, 1999, 2002; Dietrich et al. 1993, 2001; Korista et al. 1995; Kaspi et al. 2000; Shapovalova et al. 2004; Bentz et al. 2007, 2009; Sergeev et al. 2007; Popović et al. 2008; Denney et al. 2009; Denney 2010; De Rosa et al. 2015; Li et al. 2016, and references within). Studies at radio, visible, UV, and X-ray wavelengths indicate violent processes, including ejection of gas (e.g., Kollatschny \& Zetzl 2013; Kaastra et al. 2014).

According to Sergeev et al. (2007), inspection of individual broad $\mathrm{H} \beta$ profiles over a 30 -year period reveals that the broad 
emission line profiles can undergo dramatic changes (from a typical single-peaked profile centered near the systemic redshift of the galaxy, to profiles that show prominent blue or red peaks. Descriptions of blue and red peaks are presented in many papers (see e.g., Anderson 1971; Ulrich 1972; Ptak \& Stoner 1973; Peterson et al. 1987; Sergeev 1992; Shapovalova et al. 2004, 2006; Popović et al. 2008; Li et al. 2016).

AGN variability is detected at essentially all wavelengths (Netzer 2013) and on all timescales (the light-crossing time of the system, the rotational period of the central power house with the associated line-emitting gas, and the viscous time of the central accretion disk; see Czerny 2006; Netzer 2013). The light-crossing time of the BLR of more than 60 sources has been used to characterize the dimension of the system $\left(R_{\mathrm{BLR}}\right)$ and to estimate BH mass (see e.g., Kaspi et al. 2000; Gaskell \& Klimek 2003; Bentz et al. 2013; Du et al. 2015, and references therein). While most AGN vary by a factor of a few in the optical band, there have been a few examples with systematic long-term trends, like Mrk 590, which shows: (a) an overall long-term decrease by a factor of 100, along with (b) a change in Seyfert type (Denney et al. 2014), suggesting a significant decrease in accretion rate. Despite many searches for semiperiodic variations in the AGN light curves, few convincing candidates have been found so far (see e.g., Sillanpaa et al. 1988; Lehto \& Valtonen 1996; Fan et al. 1998; Rieger \& Mannheim 2000; Valtaoja et al. 2000; De Paolis et al. 2003; Sudou et al. 2003; Guo et al. 2006, 2014; Gezari et al. 2007; Bon et al. 2012; Graham et al. 2015a, 2015b; Liu et al. 2015; Shapovalova et al. 2016).

Periodic variations could be produced various ways, including binary BH systems, tidal disruption events (TDE), and more (e.g., Gaskell 1983, 2009; Sillanpaa et al. 1988; Komossa 2006, 2015; Bogdanović et al. 2008; Bon et al. 2012; Eracleous et al. 2012; Popović 2012; Valtonen \& Ciprini 2012; Bogdanović 2015; Komossa et al. 2016, and references therein). Distinguishing between scenarios requires extremely long monitoring, which is only available for a handful of sources. While AGN variability has been documented for many decades, only a few light curves span a time interval as long as 100 years to the present (e.g., NGC 4151 from 1906, Oknyanskij \& Lyuty 2007; 3C273 from the 1880s Smith \& Hoffleit 1963; and OJ287 from 1891, Valtonen \& Sillanpää 2011). Therefore, well-covered long-term light curves of nearby AGN are required to search for the presence or absence of periodic signatures. This paper presents an analysis of the very long duration light curves of NGC 5548 that span 43 years and 1600 optical spectra, including 12 years of new data. The aim of this paper is to search for periodicity in the continuum light curve, the emission line light curves, and the radial velocity curves. The structure is as follows: In Section 2 we present information about the new observations. In section Section 3 we explain our methods of calibration; in section Section 4 we discuss light and radial velocity curves. Various possible interpretations are given in Section 5. Finally, in Section 6 we summarize our results and present the conclusions.

\section{OBSERVATIONS AND DATA REDUCTION}

We analyzed 1600 spectra of NGC 5548 in the $\mathrm{H} \beta$ spectral interval, covering 43 years. We used: (a) archival spectra obtained by K.K. Chuvaev from 1972-1988 (Sergeev et al. 2007) prior to the International AGN Watch (IAW) campaigns. These early spectra were recorded on photographic plates acquired with an image tube at the $2.6 \mathrm{~m}$ Shajn Telescope of the Crimean Astrophysical Observatory. (b) The 13 year study (from 1988 to 2002) of the IAW program (Peterson et al. 2002), which provided 1530 optical continuum measurements and $1248 \mathrm{H} \beta$ measurements. $^{14}$ (c) A spectral monitoring program with the 6 and $1 \mathrm{~m}$ telescopes of the Special Astrophysical Observatory (SAO) in Russia from 1996 to 2002, and the $2.1 \mathrm{~m}$ telescope of Guillermo Haro Observatory (GHO) in Cananea, Mexico, from 1996 to 2003 (Shapovalova et al. 2004). (d) More recent, unpublished observations of the same program covering 2003-2013 observed at SAO (see Table 1), and a continuation of the monitoring campaign presented in Shapovalova et al. (2004). (e) Spectra from the new IAW campaign obtained at Asiago observatory ${ }^{15}$ in 2012 , 2013, and 2015. (f) New unpublished observations from 2013 from the Asiago observatory (also given in Table 1).

Details of the additional optical spectra obtained at INOAE and Asiago are as follows. The SAO and INOAE spectra were obtained with the 6 and $1 \mathrm{~m}$ telescopes at SAO and with the INAOE $2.1 \mathrm{~m}$ telescope at the GHO at Cananea, Sonora, Mexico. In all cases, observations were made with long-slit spectrographs equipped with CCDs. The typical wavelength range was from $4000 \AA$ to $7500 \AA$, the spectral resolution was 4.5-15 $\mathrm{A}$, and the signal-to-noise ratio $(\mathrm{S} / \mathrm{N})$ was $>50$ in the continuum near $\mathrm{H} \alpha$ and $\mathrm{H} \beta$. Spectrophotometric standard stars were observed every night. The logs of these new observations are presented in the Table 1. We also include a set of unpublished spectra that were observed with the $1.22 \mathrm{~m}$ telescope of the Asiago Astrophysical Observatory, configured in long-slit spectroscopy mode. The total exposure time was 3600 s, divided in multiple runs of 600 s or 1200 s each, to prevent the saturation of the strongest emission lines. The spectrograph used a 300 lines $/ \mathrm{mm}$ grating with a $300 \mu \mathrm{m}$ slit width, achieving a spectral resolution $R \simeq 600$ between $3700 \AA$ and $7500 \AA$. Wavelength calibration was obtained using FeAr comparison lamps, while the flux calibration was performed with the observation of the spectrophotometric standard stars Feige 34 and Feige 98. Cosmic rays were identified and masked out through the combination of the different short exposures.

\section{METHODS OF ANALYSIS}

With the goal to analyse NGC 5548 spectra from 43 years of monitoring campaigns, we performed full spectrum fitting analysis using ULySS code (Koleva et al. 2009), ${ }^{16}$ which we adopted for fitting Sy1 spectra with models representing a linear combination of nonlinear model components-emission lines, Fe II templates, AGN continuum, and the stellar population of the host galaxy. This is the first time the package was used to analyze the spectra of broad lines in AGNs. In the past it was used for (i) determining stellar atmospheric parameters using the models of stellar atmosphere $(\mathrm{Wu}$ et al. 2011) and (ii) studying the history of stellar populations (Bouchard et al. 2010; Koleva et al. 2011, 2013). Recently,

\footnotetext{
14 The IAW data can be obtained in the digital format from the following link: www.astronomy.ohio-state.edu/ agnwatch/data.html.

${ }^{15}$ With kind permission of PI Bradley Peterson to use IAW data published in Peterson et al. (2013) and unpublished data from 2012, 2013, and 2015, observed at Asiago, because there is no publication based on those data yet. ${ }^{16}$ The ULySS full spectrum fitting package is available at http://ulyss.univlyon1.fr/.
} 
Table 1

Log of the New Observations 2003-2015 from SAO, INOAE, and Asiago

\begin{tabular}{lccccc}
\hline \hline No & Date & JD-2400000 & Observatory & Aperture $(\operatorname{arcsec} \times \operatorname{arcsec})$ & Sp. Domain $(\lambda \lambda)$ \\
\hline 1 & 20030126 & 52665.924 & GHO & $2.5 \times 6.0$ & $5700-7360$ \\
2 & 20030127 & 52666.926 & GHO & $2.5 \times 6.0$ & $3800-7090$ \\
3 & 20030128 & 52667.901 & GHO & $2.5 \times 6.0$ & $3800-7090$ \\
4 & 20030327 & 52725.893 & GHO & $2.5 \times 6.0$ & $5700-7360$ \\
5 & 20030412 & 52741.582 & SAO & $4.0 \times 19.8$ & $5590-7300$ \\
88 & 20150415 & 57128.311 & ASG & $5.0 \times 6.0$ & 3.5 \\
\end{tabular}

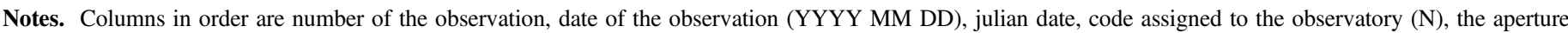
used, and the spectral region covered and the seeing.

Observatory codes:

SAO-Special Astrophysical Observatory of the Russian Academy of Science (Russia).

GHO_INOAE Guillermo Haro (Mexico).

ASG-Asiago Astrophysical Observatory (Italy).

(This table is available in its entirety in machine-readable form.)

Bon et al. (2014) tested the accuracy of the code in recovering stellar population and gas parameters in Type 2 AGNs.

We obtained light curves and radial velocity curves for all spectra and searched for possible periodicities. We used standard methods for treating unevenly spaced data, and a new method that developed for our specific conditions of very few cycles and the very specific sampling characteristics of the obtained data series (see Section 4.1).

\subsection{Line and Continuum Fittings}

Because ULySS gives us a choice of defining and including components, we adjusted it to simultaneously analyze every component that contributes to the flux in the wavelength region around $\mathrm{H} \beta$. For analyzing the variability of NGC 5548, we defined the model $M(x)$ as follows:

$$
\begin{aligned}
M(x)= & P(x)([T(x) \otimes G(x)]+C(x)+N(x) \\
& \left.+B(x)+\sum_{i=1}^{4} F e \mathrm{II}_{i}(x)+\sum_{j=1}^{n} S_{j}(x)\right),
\end{aligned}
$$

where $M(x)$, represents a bounded linear combination of nonlinear components-stellar template spectrum $T(x)$ convolved with a line of sight velocity broadening function, an AGN continuum model $C(x)$, a sum of narrow $N(x)$, and semibroad components $B(x)$ of [O III] emission lines, respectivelyas well as a sum of Gaussian/Gauss-Hermit functions $\mathrm{S}(x)$ accounting for other AGN emission lines in the analyzed spectral domain and the Fe II template consisting of four groups of Fe II lines.

A multiplicative polynomial $P(x)$, which represents a linear combination of Legendre polynomials, was included in a fit to eliminate the overall shape differences between the observed stellar and galactic spectra. The introduction of this polynomial ensures that the results are insensitive to the normalization, Galactic extinction, and the flux calibration of a galaxy and stellar template spectra (Koleva et al. 2008). For simplicity, we assumed a Gaussian velocity broadening function $\boldsymbol{G}(\boldsymbol{x})$, but it is also possible to use the Gauss-Hermite polynomials (Rix \& White 1992; van der Marel 1994). The contribution of the components to the total flux can be obtained from their weights, which are determined at each Levenberg-Marquardt (Marquardt 1963) iteration using a bounding value least-square method (Lawson \& Hanson 1995).
For the stellar population model we used grid of PEGASE. HR single stellar populations, computed with the Elodie.3.1 library and a Salpeter IMF (Le Borgne et al. 2004). The model $M(x)$ is generated at the same resolution and with the same sampling as the observation, and the fit is performed in the pixel space. The fitting procedure performs the LevenbergMarquardt minimization (Marquardt 1963). In modeling the integrated spectra of NGC 5548 we added the power law to the stellar population base to represent an AGN featureless continuum $\left(f_{\lambda} \sim \lambda^{\alpha}\right)$. The spectral index $\alpha$ depends on the continuum slope, and represents the free parameter in the fit. To tie the parameters of the [O III] lines, we defined two separate components of the model: a narrow component and a semibroad component. In this way we tied the widths, shifts, and intensities of [O III] components (the intensity ratio was kept to $3: 1)$. The rest of the emission lines in the domain $\lambda \lambda[4430,6400]$ were fitted with a sum of Gaussians; we fitted He II $4686 \AA$ with two components, and used four components in the fit of $\mathrm{H} \beta$ line: narrow, broad blueshifted, broad redshifted, and very broad component. As proposed by Kovačević et al. (2010), we defined four groups of Fe II lines to fit the $\mathrm{Fe}$ II multiplets around the $\mathrm{H} \beta$ line: Fe II s, p, and $\mathrm{f}$ group, and I Zw1 template. Because it is very difficult to fit the stellar population in the spectra of Sy1 galaxies with broad emission lines, in the first step we fitted the spectra of NGC 5548 in the minimum of activity with all free parameters of the model (defined with Equation (1)), and with multiplicative polynomials of the 15th order (as in Bon et al. 2014). We find that a single stellar population with an age of $7200 \mathrm{Myr}$ and metallicity $[\mathrm{Fe} / \mathrm{H}]=0.2$ fits the best spectra in the minimum. In fitting the rest of the spectra from monitoring campaigns of this galaxy, we fixed age and metallicity at best-fit values from the first step, and used the multiplicative polynomial of the first order to minimize the effects of this polynomial on the fit of emission lines. Free parameters associated with the stellar population were kinematic parameters-mean stellar velocity and dispersion. Examples of best-fit spectra at the minimum and maximum activity are presented in Figure 1.

All the results presented below are based on the assumption that the luminosity of the [O III] 5007 line, and all other narrow emission lines, does not change in time (see for example Peterson et al. 2002; Shapovalova et al. 2008). We assumed that the slit width and position angles used in the various campaigns were not affected by the O III region size in the 
(a)

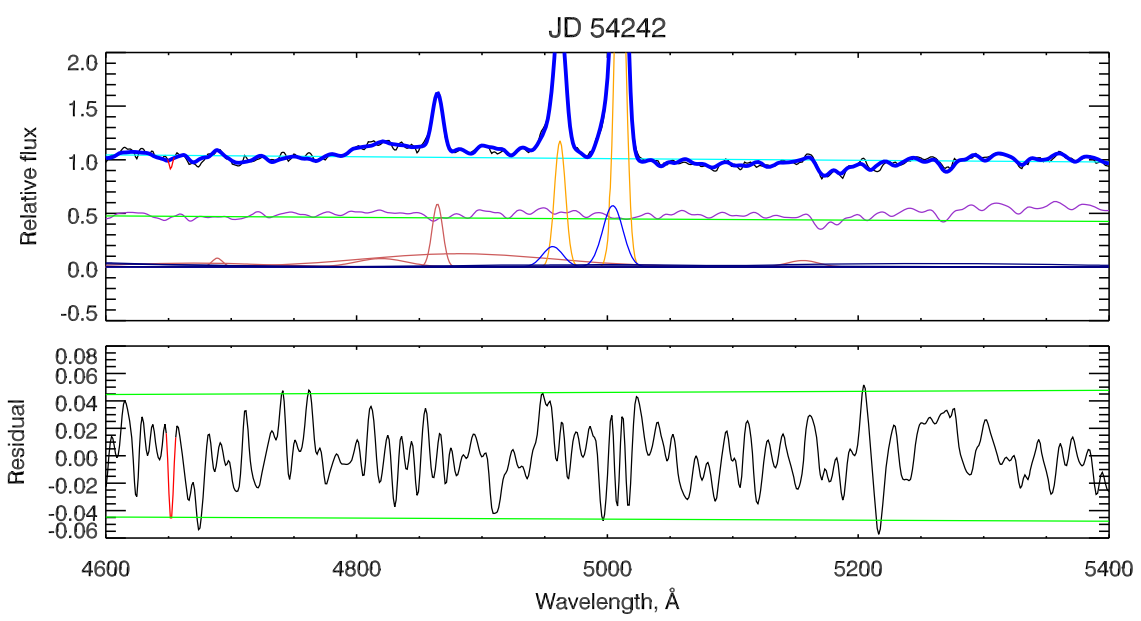

(b)

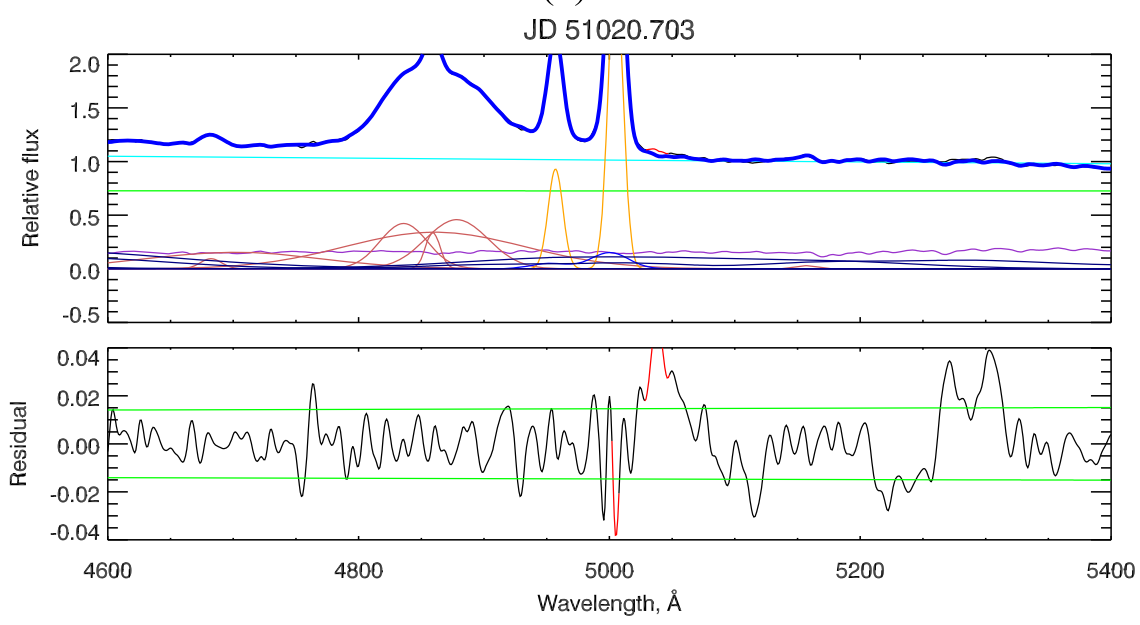

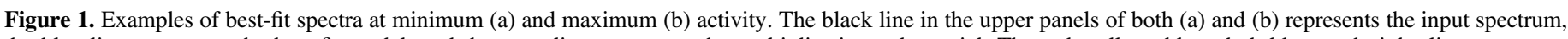

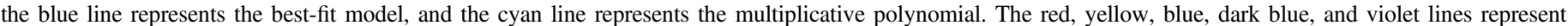

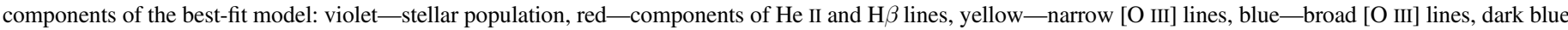

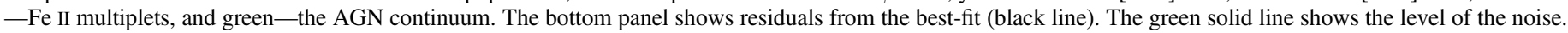

NGC 5548, which appeared to be very compact (see Schmitt et al. 2003). For that reason we made a simple test to estimate light loss for smallest slit used (see the Appendix). The spectra were scaled to a constant flux of $\mathrm{F}([\mathrm{O} \mathrm{III}] \lambda 5007)=5.58$. $10^{-13} \mathrm{erg} \mathrm{s}^{-1} \mathrm{~cm}^{-2}$, and we constructed host galaxy subtracted continuum light curves of continuum flux measured at $5100 \AA$ and the broad $\mathrm{H} \beta$ emission line. The $5100 \AA$ continuum light curve and the $\mathrm{H} \beta$ light curve are presented in Figure 2.

From the set of 1494 "IAW" spectra, we remove 128 spectra of the $\mathrm{H} \alpha$ region only, 109 spectra with poor spectral resolution, and 48 spectra that did not cover a large enough range to measure the $5100 \AA$ continuum. This left 1209 IAW spectra, which combined with 249 spectra from Crimean Astrophysical observatory, 83 spectra from $\mathrm{SAO} / \mathrm{GHO}$ observatories, and 7 spectra from Asiago, totaled to 1548 spectra. After careful inspection of the fits, we removed 10 additional spectra, leaving 1538 for further analysis (Table 2).

The zero-point error in the radial velocity was measured from the scatter of the difference between the peak, $v_{r}$ of [O III] $\lambda 5007$ and the narrow component of $\mathrm{H} \beta, \delta v_{r}=v_{r}(\mathrm{H} \beta)-v_{r}(\mathrm{O}$ III $) \approx$ $35 \mathrm{~km} \mathrm{~s}^{-1}$. We note that there is a known small systematic offset of $\delta v_{r} \approx+19 \mathrm{~km} \mathrm{~s}^{-1}$, frequently found in [O III]-strong AGN (e.g., Eracleous \& Halpern 2003; Hu et al. 2008; Komossa et al. 2008; Marziani et al. 2016, and references therein). The velocity zero point was set on the peak wavelength of [O III] $\lambda 5007$, because [O III] $\lambda 5007$ is a very sharp feature of high $\mathrm{S} / \mathrm{N}$ and is less influenced by the underlying broad $\mathrm{H} \beta$ profile.

Typical errors on light and radial velocity curves were estimated from the dispersion of the measured parameters on short time intervals ( $<20$ days), in some cases multiple spectra were available (five for the continuum light curve). Because $20 \mathrm{~d}$ is a period that is long enough to include possible significant intrinsic variations, we considered the time behavior of the minimum dispersion value around the average of a given parameter (for example, FWHM, HWs) computed over $20 \mathrm{~d}$ as a function of time. Typical rms errors for $\mathrm{H} \beta$ seem to be around $5 \cdot 10^{-13} \mathrm{erg} \mathrm{s}^{-1} \mathrm{~cm}^{-2}$, while for the typical rms scatter for the $5100 \AA$ continuum flux is $10^{-16} \mathrm{erg} \mathrm{s}^{-1} \mathrm{~cm}^{-2} A$.

Li et al. (2016) discussed a large part of a similar dataset in a somewhat similar way. However, the main differences between Li et al. (2016) and our analysis was in our unified approach and the quantity of data used. In our study this was achieved 

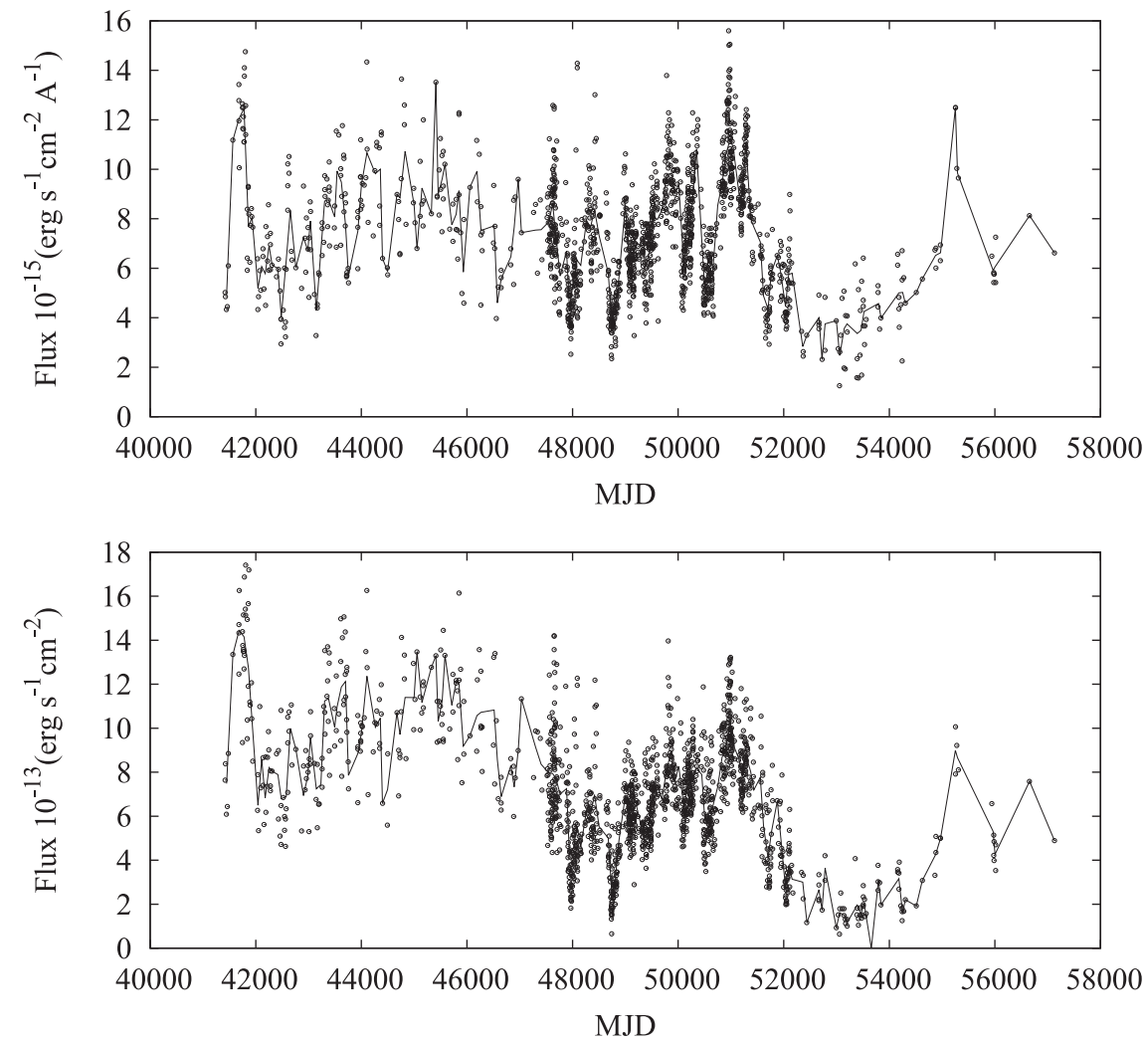

Figure 2. Top: continuum $5100 \AA$ light curve (dots) and 80-day binned continuum light curve (slashed line). Bottom: same as in the top panel, but for H $\beta$.

using a more robust method and taking into account some important components that were not considered by $\mathrm{Li}$ et al. (2016; i.e., the galactic host emission, Fe II multiplets, He emission, and two components of each [O III] narrow line in spectral fit). As mentioned above, 1538 spectra were analyzed (with the new spectra covering the last 12 years), instead of only about 850 used by Li et al. (2016), with a large gaps in the last 12 years of their time series.

\section{RESULTS}

\subsection{Variability Analysis}

Light and radial velocity curves were analyzed for possible periodicity using standard methods, such as Lomb-Scargle (Lomb 1976; Scargle 1982), as well as the new method for unevenly sampled data tailored to the specific special conditions of our data series. In addition to light curves, we constructed curves using measurements of different fractional intensities of blue side (blue dots) and red side (red dots) $\mathrm{H} \beta$ broad emission line at $25 \%, 50 \%, 75 \%$, and $90 \%$ as a function of time, which behave as half-width radial velocity curves at these fractional intensities. We also calculated line centroids, ${ }^{17}$ and full widths. ${ }^{18}$ Half widths and centroid radial velocity curves are presented in Figure 3. Full widths are also calculated, and their curves are presented in Figure 4. We applied different methods to test for possible periodicities using a similar method as for light curves.

\footnotetext{
${ }_{17}$ Centroids are calculated as $\lambda_{c}=\left(\lambda_{\text {red }}+\lambda_{\text {blue }}\right) / 2$.

${ }^{18}$ Full widths are calculated as $\lambda_{\mathrm{FW}}=\left(\lambda_{\text {red }}-\lambda_{\text {blue }}\right)$.
}

\subsection{Lomb-Scargle Periodicity Analysis}

Using the Lomb-Scargle (Lomb 1976; Scargle 1982) analysis (LS method), we analyzed light and radial velocity curves with previously removed linear trends. Results can be seen in Table 3.

To avoid very different sampling, we analyzed rebinned curves. This also eliminated shorter variations that correspond to the light-crossing timescale of the system. We rebinned the radial velocity curves to the 80 -day average bins because the variability lags of $\mathrm{H} \beta$ to continuum variations were less than 30 days (Kaspi et al. 2000; Peterson et al. 2002; Zu et al. 2011; Koshida et al. 2014). According to Czerny et al. (1999), there are two clearly distinct physical mechanisms of variability in NGC 5548 curves with two different timescales: one less than 30 days and another with an order above 100 days. The short timescale variability is connected to the Comptonization of the soft photons emitted by the innermost part of the accretion disk, while in the long timescales the optical variability is not related to the X-rays (see more in Czerny et al. 1999). Therefore, we assumed that 80-day binning would be long enough to filter short variations and only analyze the longer ones. We found periodicities with very low false alarm probability in the radial velocity curves of half widths measured at $25 \%, 50 \%, 75 \%$, and $90 \%$ of $\mathrm{H} \beta$ line maximum (see Table 3). The obtained periodicities show similar values. We also, searched for periodicity in the radial velocity curves of the full widths at $25 \%, 50 \%$, and $75 \%$ of the maximum intensity of the broad $\mathrm{H} \beta$ emission line. The results are presented in Figure 5. There is a significant peak at about 3000 days, which is about half the value detected at half widths radial velocity curves (see Table 3). As argued below, these periodicities are far from being sinusoidal and cover a very small number of repeating 

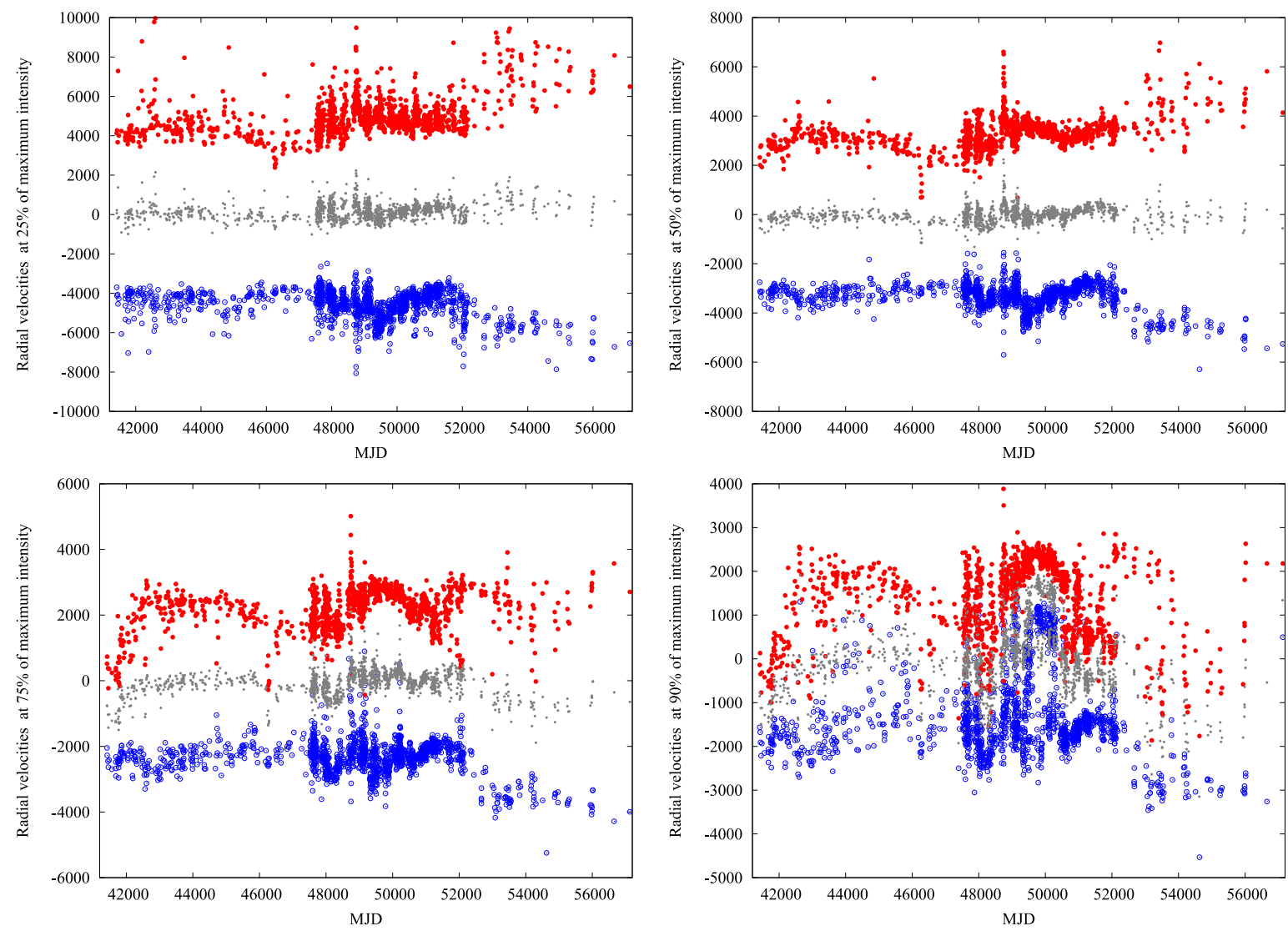

Figure 3. Radial velocities measured on the blue (open circles) and red (filled circles) sides of $\mathrm{H} \beta_{\mathrm{BC}}$ at fractional intensities of $25 \%$ (top left), 50\% (top right), $75 \%$ (bottom left), and $90 \%$ (bottom right). Centroids (gray dots) are calculated as the averaged value of the blue and red side fractional intensity radial velocity measurements.

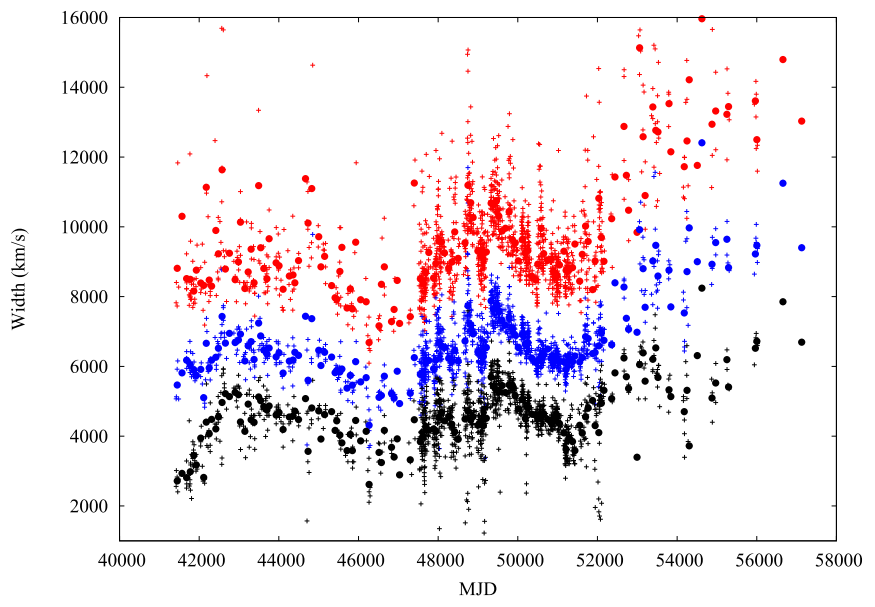

Figure 4. Curves of the full width at different heights of the broad $\mathrm{H} \beta$ line in A: FW25\% (red), FW50\% (blue), and FW75\% (black). The observations are presented with crosses, while the binned averaged data are plotted with full circles.

periods (a little less then 3). We suspect that the standard LS analysis may not be reliable enough in such cases, and so searched for a more robust method that is more suitable for this type of data. The method is explained below and the numbers listed are based on the standard LS method and should be regarded as "tentative periods." We note that the LS method is known to give spuriously high significance levels to lowfrequency periods for "red noise" variability (see Westman et al. 2011; Vaughan et al. 2016; N. Bon et al. 2016, in preparation).

It is interesting to note that red and blue side radial velocity curves at $25 \%$ and $50 \%$ are anticorrelated, while red and blue radial velocity curves at $90 \%$ show a positive correlation (Figure 6). As we go toward the top of the line, the anti correlation switches to correlation (see Figure 6). This indicates that the peak of the line is shifting with the red side of the line, while at the base of the line radial velocity curves could be affected by two different kinematic components.

To analyze the $\mathrm{H} \beta$ line shifts we also obtained a single Gaussian fit of the spectra and constructed a radial velocity curve from the obtained shifts. We fit a sine function, assuming the expected periodicity of 5700 days (see Figure 7), to lead the eye and not to claim a simple sinusoidal periodicity, because there are obvious deviations from the sinusoidal curve in several epochs. One can see some similarity of this radial velocity curve and the one obtained from measurements of red half width at $75 \%$ of the line maximum (see Figures 7 and 3), implying that the line shifts are mainly affected by variations on the red side of the line. Also, the similarity of these curves could indicate the same periodicity.

\subsection{A New Method for Finding Periodicity in Unevenly Sampled Data}

As explained, our data are obtained from many different monitoring campaigns with very different sampling patterns. From the light curves one can see that the amount of data is much higher in the second third of the observed interval than 
Table 2

Spectral Parameters Obtained from the Best Fits

\begin{tabular}{lccccccccrrrr}
\hline \hline D-2400000 & Flux Hb & Err. & Flux 5100 & Err. & B25\% & R25\% & B50\% & R50\% & B75\% & R75\% & B90\% & R90\% \\
\hline 41420.546 & 7.64 & 0.14 & 5.02 & 0.08 & -3695.2 & 4127.0 & -2750.7 & 2317.6 & -2040.4 & 518.9 & -1565.9 & -137.8 \\
41426.531 & 8.38 & 0.16 & 4.86 & 0.09 & -4131.3 & 4272.3 & -3116.2 & 2012.3 & -2277.5 & 737.1 & -1677.0 & 131.7 \\
41446.471 & 6.10 & 0.02 & 4.33 & 0.01 & -4065.1 & 3665.6 & -3169.5 & 2749.3 & -2451.0 & 379.8 & -1911.0 & -345.5 \\
41460.463 & 6.44 & 0.18 & 4.44 & 0.13 & -4542.0 & 7289.4 & -3349.8 & 2805.7 & -2632.2 & -227.5 & -2212.7 & -770.2 \\
41484.456 & 8.85 & 0.15 & 6.10 & 0.05 & -4265.2 & 4006.7 & -3131.6 & 1932.5 & -2413.4 & 355.8 & -1933.6 & -308.7 \\
41566.266 & 13.35 & 0.42 & 11.18 & 0.02 & -6064.2 & 4237.7 & -3640.7 & 2173.0 & -2689.5 & 242.6 & -2153.1 & -418.1 \\
41681.662 & 14.71 & 0.73 & 11.96 & 0.40 & -5332.9 & 3892.3 & -3795.3 & 2435.8 & -2904.6 & 384.4 & -2368.8 & -516.2 \\
57127.811 & 4.90 & 0.10 & 6.62 & 0.07 & -6528.3 & 6501.3 & -5263.4 & 4137.5 & -3993.0 & 2702.0 & 496.7 & 2181.6 \\
\hline
\end{tabular}

Note. Table lists modified julian date (N1), $\mathrm{H} \beta$ flux in units $10^{-13} \mathrm{erg} \mathrm{s}^{-1} \mathrm{~cm}^{-2} \AA^{-1}$, continuum flux measured at $5100 \AA$ in units $10^{-15} \mathrm{erg} \mathrm{s}^{-1} \mathrm{~cm}^{-2}$, and fractional intensity of blue and red side widths measured at $25 \%, 50 \%, 75 \%$, and $90 \%$ of maximum intensity of broad $\mathrm{H} \beta$ emission line. Julian dates of IAW data in the form “*.1" are used to artificially differentiate between two different spectra under the same mjd on the IAW website (e.g., 48325.0 and 48325.1 ).

(This table is available in its entirety in machine-readable form.)

the first and third parts; the latter contain less than a few hundred observations each, while the former contained above a thousand spectra. This distribution introduces various biases in standard methods for periodicity analysis of unevenly sampled data, based on sine function decomposition such as Fourier and Lomb-Scargle. The Lomb-Scargle method (LS) is commonly used to detect periodicities in unevenly sampled time series. LS follows the approach of Fourier methods and fits a sinusoid to the data. However, in classic Fourier methods uniform sampling gives rise to the orthogonality properties of the trigonometric functions, which has profound statistical implications. Thus, the Fourier series terms can be shown to be statistically independent under very broad conditions. This is not the case in LS unevenly sampled data. Nevertheless, it is a common practice to interpret the LS periodogram as a kind of Fourier series, relying on the assumption that the uneven sampling times are uniformly distributed. It is possible to neglect this effect if many cycles are included in the analyzed timespan. However, in our case the sampling has an obvious nonuniform nature, where the middle part of the time series is sampled much more densely than the rest. Moreover, we focus our attention on long periods, meaning only two to three cycles in total. Thus, the simplifying assumptions that allow the use of LS are no longer valid, and we must tailor a method to take into account the nature of the specific sampling pattern. Comparing Table 2 and Figure 5 shows that even though Lomb-Scargle analysis gives a very high probability of periodicities, the values of the obtained periods are dispersed. The periodicity result values span between 5500 and 6200 days. Very unevenly sampled data clearly require a method that can recognize repeating patterns, not only sine functions (as in cases of Fourier or Lomb-Scargle analysis). For that purpose we proposed method tailored to this specific case, with the special conditions of very few cycles and specific sampling characteristics.

We started from the working assumption that the data between JD $=47508$ and JD $=52175$ are well sampled and of better quality than the rest (see e.g., Figure 2). Those two problems prevent proper use of conventional period search techniques, because they usually assume many cycles and homogeneous data quality and sampling. Our new method treats the best-sampled part as fixed, and tests only the noise and sparsely sampled parts on either end to see how well they fit the hypothesis of a period. We first linearly interpolate the
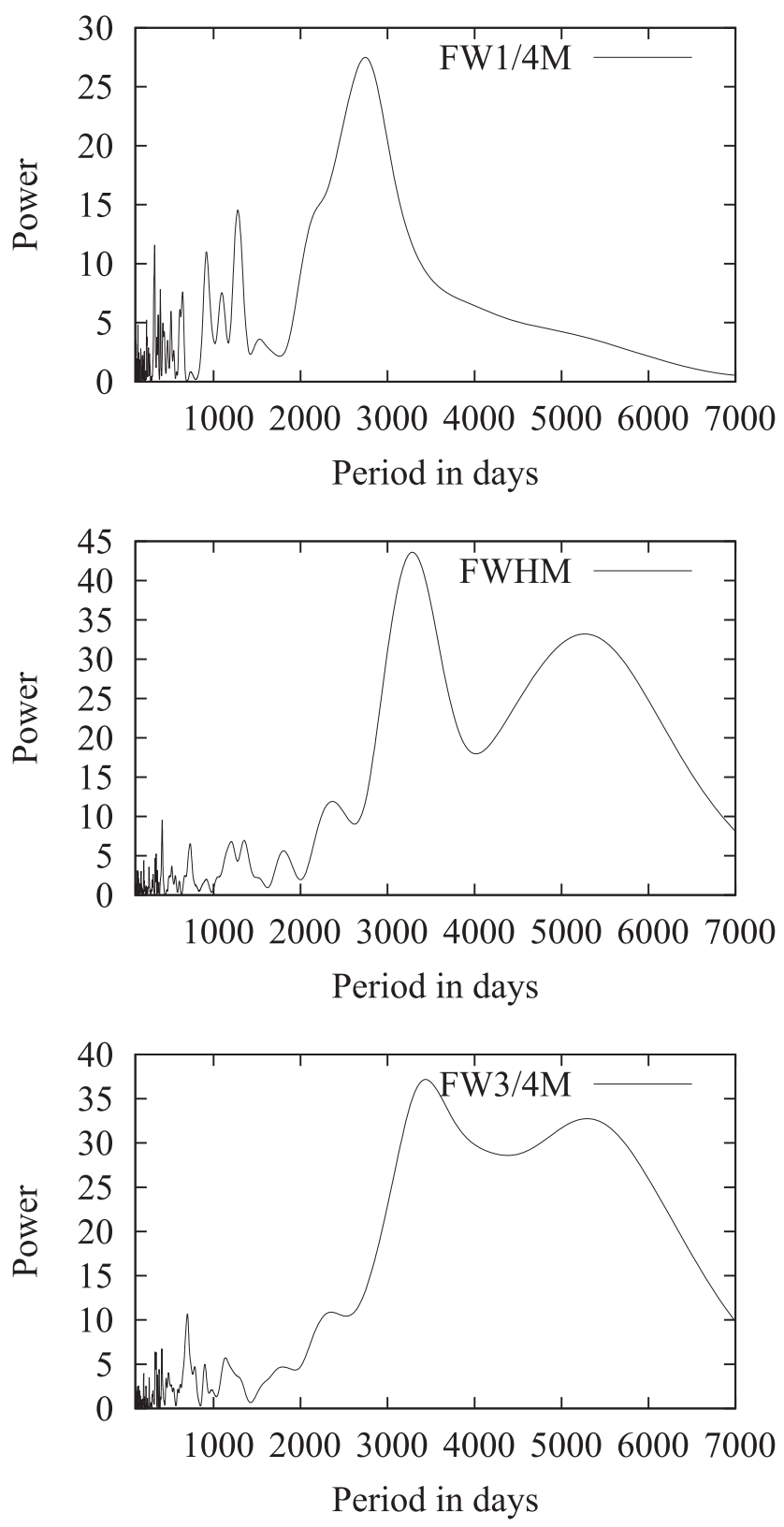

Figure 5. Lomb-Scargle periodograms of radial velocity curves of full widths at $25 \%$ (top panel), $50 \%$ (middle panel), and $75 \%$ (bottom panel). 
Table 3

Results from the Periodicity Analysis on Light Curves of Continuum at $5100 \AA$ and $\mathrm{H} \beta$ Fluxes, and Radial Velocity Curves of Half Widths of Broad $\mathrm{H} \beta$ Emission Line Measured at Blue and Red Side of $25 \%, 50 \%, 75 \%$, and $90 \%$ of Maximum Intensity

\begin{tabular}{lcc}
\hline \hline Curve: & L-S & $p$-val. \\
\hline LC C. 5100 A & 5490 & $<10^{-7}$ \\
LC H $\beta$ & 5710 & $<10^{-8}$ \\
RV 25\% blue & 5798 & $<10^{-8}$ \\
RV 25\% red & 5670 & $<0.09$ \\
RV 50\% blue & 5920 & $<10^{-8}$ \\
RV 50\% red & 5730 & $<10^{-2}$ \\
RV 75\% blue & 5740 & $<10^{-8}$ \\
RV 75\% red & 5740 & $<10^{-8}$ \\
RV 90\% blue & 5950 & $<10^{-8}$ \\
RV 90\% red & 6240 & $<10^{-7}$ \\
FW50\% & 2960 & $<0.002$ \\
FW25\% & 3430 & $<10^{-8}$ \\
FW75\% & 3302 & $<0.0005$ \\
\hline
\end{tabular}

Note. In the table are the given period and the false alarm probability ( $p$-value) for the Lomb-Scargle periodogram (Lomb 1976) of 80-day binned data series, where we present longest significant periods.

best-sampled part. Then we produce a periodogram by trying many periods. For each trial period we fold every point we test into the best-sampled part, and calculate its deviation from the interpolation (Similar to the phase dispersion minimization method; see Stellingwerf 1978; Plavchan et al. 2008). We sum those squared deviations and obtain a significance score that is similar to the $\chi^{2}$ score for the periods. If there is a periodicity it should come up as a lower than the usual value of this score.

We look for a minimum (it is basically a $\chi^{2}$, but without normalization by the errors), and found it at a period of 5676 days (see Figure 8). But we still need to test how statistically significant the minimum is. To do this we consider the central segment as a reference we do not change, and test whether the points in the first and third segments fit the central part after phase folding. For this we carry out the following permutation test. In each iteration we randomly reshuffle the values of the points in the first and third segments (the measurements, not the sampling times). Thus we "ruin" their time dependency, but keep their "window function." We then recalculate the periodogram, and look for the minimum value. This determines the fraction of random reshufflings that will produce a value lower than the value obtained with the actual light curve. Only 2 out of 10,000 values were lower, which means the results have a significance $p$-value of around 0.0002 .

The Figure 8 shows the actual periodogram (in black) against the background of 1000 periodograms out of the 10,000. The structure of the problem imposes some structure on the periodograms, but the result stays significant because the 5676 periods the $\chi^{2}$ trough look quite displaced from their randomly produced counterparts.

We repeated the analysis on the radial velocity curves shown in Figure 3, and show the results in the bottom panel of Figure 8 . The periodicity looks very significant on the radial velocity curve measured at red side $75 \%$ of the line maximum; the lowest point (i.e., best fit) is at a period of 5725 days, which is essentially identical to the $\mathbf{5 6 7 6}$ day period in the flux. As judged from the new method, none of the other radial velocity curves shown in Figure 3 shows a statistically significant periodicity.
It is important to emphasize that real radial velocity periodicities may be present in the $\mathrm{H} \beta$ profile, but the noisy half-width radial velocity curves do not allow us to detect them unambiguously.

If we assume that the periodic component is sine-like with a periodicity of 5676 days, the variance (see e.g., Nandra et al. 1997; Nikolajuk et al. 2004) of the data carried by the periodic component (with a secular trend) is about $19 \%$. We consider both the variance in the data and the secular component under the assumption of long timescale (15.7 years) sinusoidal variations.

\section{POSSIBLE INTERPRETATIONS}

The main results of our investigation are the detections of significant periodicities in the the luminosities of the $5100 \AA$ continuum and $\mathrm{H} \beta$ light curve, as well as in the radial velocity curve of the $\mathrm{H} \beta$ profile. The periods are very similar and consistent with $P \approx 5700$ days ( $\approx 15.6$ years; see Table 2 ). This value is practically identical to the period found in the supermassive binary black hole (SMBBH) candidate NGC 4151 (15.8 years; see Oknyanskij 1978; Bon et al. 2012) and similar to 11-year period of OJ287 (Valtonen \& Ciprini 2012). It is about twice the periodicity found for another recently found supermassive binary candidate, PG 1302-102 (Graham et al. 2015a). Our best measured periodicity is similar to the 14year periodicity found recently by $\mathrm{Li}$ et al. (2016) for NGC 5548, but our result is based on a more robust analysis and more data. In this section we briefly discuss several possible physical scenarios that can give rise to the observed periodicity. We focus on the more secure results obtained with the new method of simulations presented in Section 3. We consider these results to be the most reliable, and the ones obtained with the LS method more questionable. This does not exclude the possibility that some of the LS results, such as the suggested periodicity of the FWHM velocity curve, are not part of the suggested scenario. In every model we assume a $\mathrm{BH}$ mass based on the results of multiple reverberation mapping campaigns. We adopt $5.73_{-0.24}^{+0.25} \times 10^{7} M_{\odot}$, as given in the $\mathrm{BH}-$ mass database of Bentz \& Katz (2015), and our own estimate of the normalization factor $f \approx 3.75$ used in the expression $M_{\mathrm{BH}}=f \sigma_{\mathrm{rms}}^{2} r_{\mathrm{ct}} / G$ with the velocity dispersion computed from the rms spectrum. This mass differs by only $30 \%$ from the mass given by Bentz et al. (2007), who assumed $f=5.5$ and $6.3 \times 10^{7} M_{\odot}$. A circular orbit around such a BH would have a period $P \approx 17.5 r_{15 \mathrm{ld}}^{\frac{3}{2}} \times M_{5.710^{7}}^{-\frac{1}{2}}$ years, where $r=15 \mathrm{ld}$ is the radius in units of $15 \mathrm{ld}$. This reference radius was chosen because it is typical of the many annual means of the lags of $\mathrm{H} \beta$. Because reverberation time delays vary within $6 \mathrm{ld}$ and 27 ld (Bentz \& Katz 2015), the expected periods are between 8 and 36 years. Obviously, the reverberation mapping results give a responsivity-weighted radius and the total line emission from a wider range of radii.

The $\mathrm{H} \beta$ reverberation mapping distances can be compared with the reverberation measurements of the inner radius of the dust torus, which range between 40 and 80 ld depending on the observing season and delay computation techniques (Koshida et al. 2014). Finally, because some of the models we discuss in this section involve a second $\mathrm{BH}$, we refer to the $5.7 \times 10^{7} M_{\odot}$ $\mathrm{BH}$ as the primary $\mathrm{BH}$ in the system. 

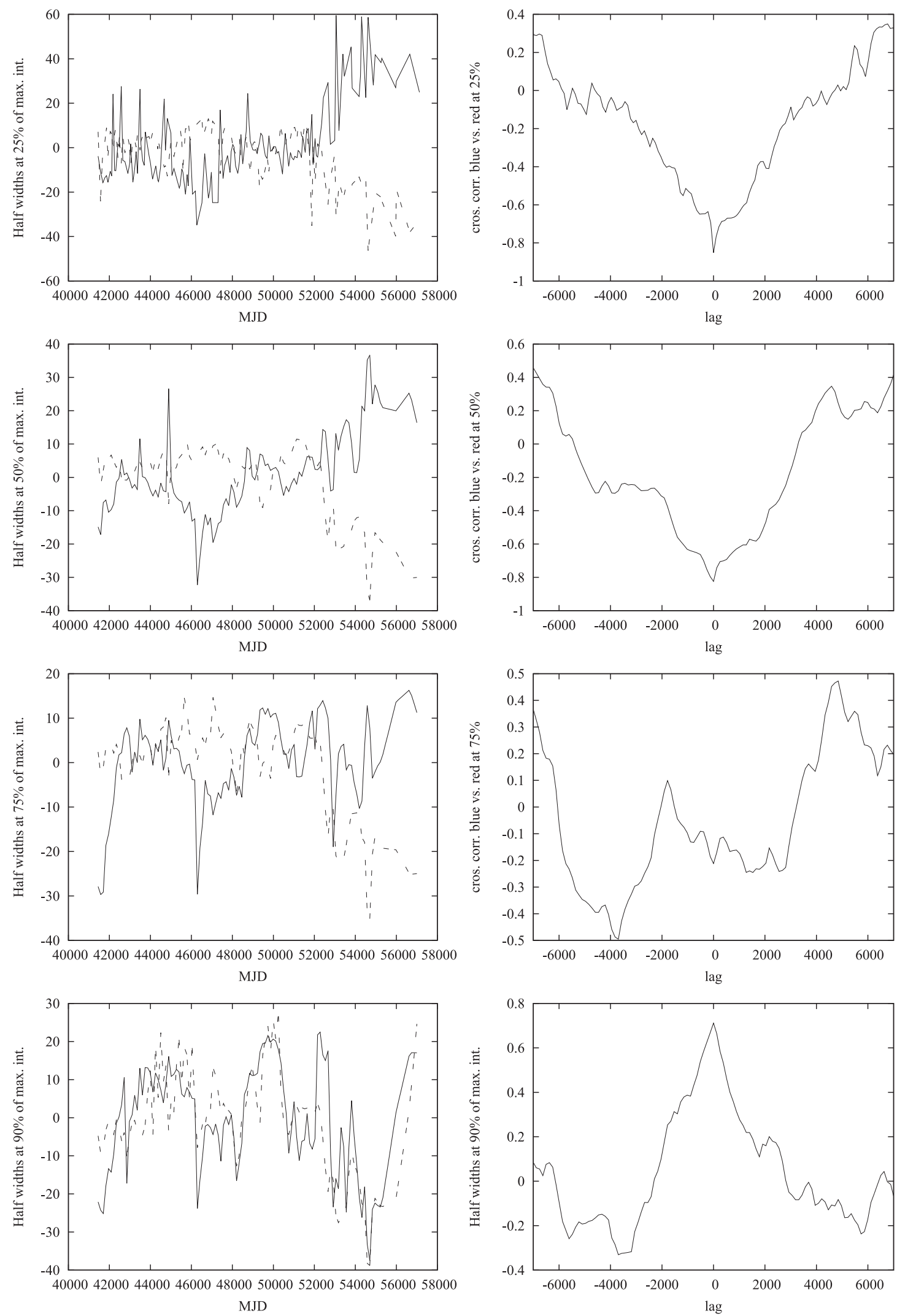

Figure 6. Left: radial velocity curves measured as red side width (thick line) and blue side width (slashed line) of the broad $\mathrm{H} \beta$ emission line at $25 \%, 50 \%, 75 \%$, and $90 \%$ of the line maximum, rebinned to 80 days with mean value subtracted and overplotted to show shape similarity. Right: cross-correlation functions between corresponding pairs of curves. The cross-correlation functions of the red and blue side radial velocity curves at $25 \%, 50 \%$, and $75 \%$ broad $\mathrm{H} \beta$ emission line are negative (anticorrelated), while at $90 \%$ they are correlated. This indicates that the peak of the line measured on the blue and red side on $90 \%$ is shifting as a single component, while at the base of the line, radial velocity curves could be affected by two oppositely moving components. 


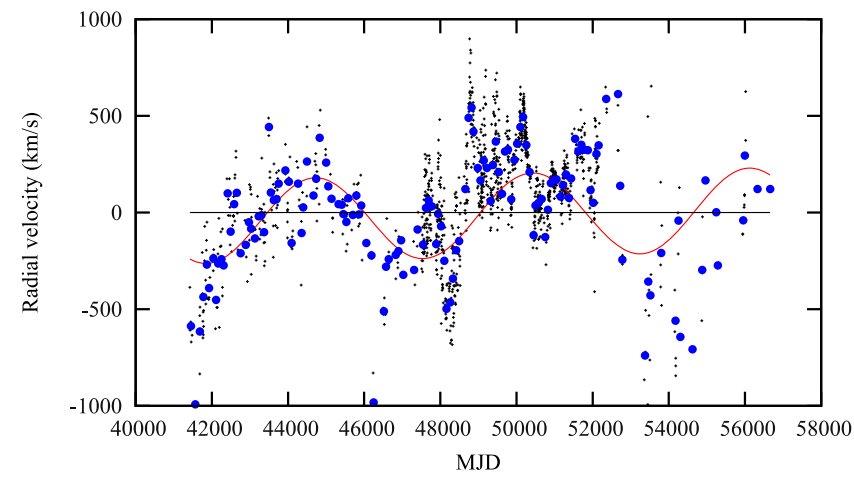

Figure 7. Radial velocity curves resulting from fitting a Gaussian to the broad $\mathrm{H} \beta$ line of NGC 5548, as discussed in the text. The solid red line shows the best fit of a sine wave of period 5700 days.
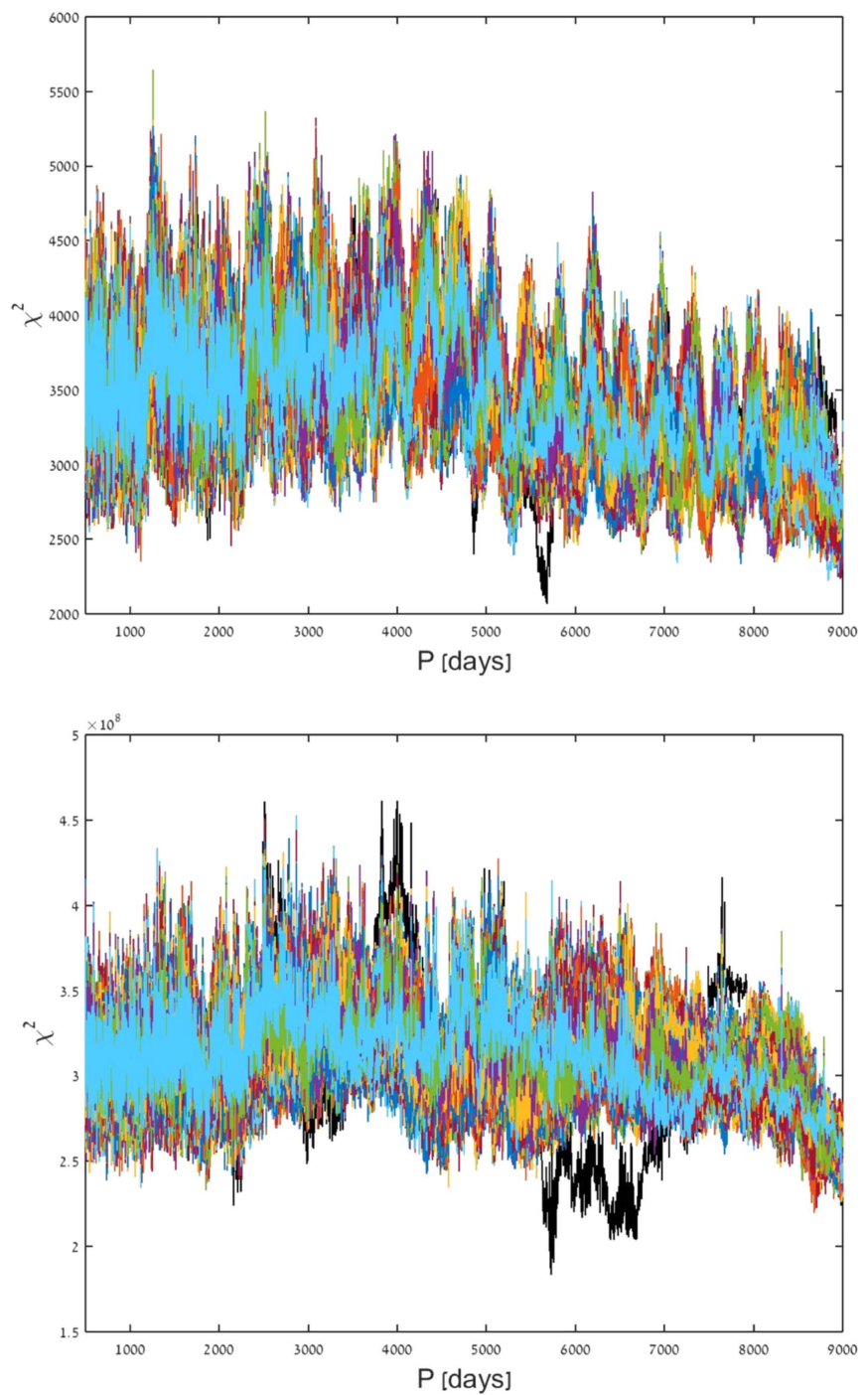

Figure 8. Periodogram of the 1000 permutations for the continuum light curve at $5100 \AA$ (top) and a radial velocity curve measured at $75 \%$ of the maximum line intensity at the red side of $\mathrm{H} \beta$ (bottom).

\subsection{Geodetic Precession, Disk-self Warping, Hot Spots and Spiral Arms}

We can readily exclude two mechanisms that could give rise to a significant periodicity in a single $\mathrm{BH}$ system, as discussed in Bon et al. (2012): geodetic precession and disk-self warping induced by radiation pressure (Pringle 1996). The first of these occurs on timescales that are in general much longer than the observed periodicity (Begelman et al. 1980). As for the latter, Graham et al. (2015a) showed that warped disks around single $\mathrm{BHs}$ are not favored in an AGN context. In particular, the expected periodicity in such a scenario is much longer than the period found for NGC 5548 (e.g., a BH mass of $10^{8} M_{\odot}$ gives a period between $10^{2.2-6.9}$ years; Pringle 1996; Graham et al. 2015b). Mechanisms that could produce orbital periodicity are reperturbation in a very large central disk that extends far beyond the inner accretion disk whose dimensions are known from recent reverberation mapping to be of order 2 ld (De Rosa et al. 2015; Edelson et al. 2015; Fausnaugh et al. 2016). A hot spot rotating at a distance of about $15 \mathrm{ld}$, which is not observed during a fraction of the orbit, can explain the periodic continuum variations. Emissivity perturbation by spiral arms in a large central disk can result in a double-peaked profile and emission line shape variability (see Chakrabarti \& Wiita 1994; Jovanović et al. 2010; Lewis et al. 2010). Spiral arms can be triggered by the close passage of a massive object, such as a massive star cluster or super massive BH (see Lewis et al. 2010, and references within), by gravitational instabilities (see e.g., Flohic \& Eracleous 2008, and references therein), or by an object passing through the extended disk (Chakrabarti \& Wiita 1993). Fragmented spiral arms can account for emission line shape variation on relatively short time and radial velocity changes occurring on the dynamical timescale (Jovanović et al. 2010; Lewis et al. 2010). Such substructures in a nonuniform central disk could cause excess emission moving across the line profile (Jovanović et al. 2010; Lewis et al. 2010; Goosmann et al. 2014). We do not consider such models to be plausible explanations for NGC 5548 because of the huge central disk that is not observed in this source (the hot spot that, to explain the periodic variation should emit a sizable fraction of the luminosity of the small inner disk), and because the overall emission line spectrum of NGC 5548 is very similar to those observed in thousands of AGN of similar luminosity; these scenarios cannot explain all the population properties.

\subsection{Tidal Disruption Events}

A TDE when a star is disrupted by a BH creates an appearance somewhat like an AGN for a limited duration. The tidal radius (i.e., the distance from the $\mathrm{BH}$ at which a star is tidally disrupted) can be written as $r_{t} \approx 1.5 \times 10^{13}\left(M_{\mathrm{BH}, 7}\right)^{\frac{1}{3}}\left(M_{\star, \odot}\right)^{\beta} \mathrm{cm}$ (Hills 1975; Komossa 2015), where $M_{\mathrm{BH}}$ is the mass of the $\mathrm{BH}$ in units of $10^{7} M_{\odot}$, and the mass of the star is in solar mass. The exponent $\beta$ is $\approx 2 / 3$ or $1 / 6$ depending on whether the star is a main sequence star $M_{\star}$, has mass $\lesssim 1 M_{\odot}$, or belongs to the upper main sequence with $M>2 M_{\odot}$ (Torres et al. 2010). For the NGC $5548 \mathrm{BH}$ mass and for a main sequence star, the tidal radius is extremely small: a few gravitational radii. A main sequence star may therefore orbit at 2-15 ld without suffering a TDE. In the absence of a pre-existing accretion disk, tidal disruption causes a luminous flare with a short rise and a longer-lasting decline (e.g., Rees 1990), as observed in several cases (e.g., Komossa \& Bade 1999; Gezari et al. 2012, and references therein). A partially stripped star in an orbit around the SMBH can cause repeated accretion events each time its orbit passes near pericenter, and may thus produce a semi-periodic signal in the light curve (e.g., Hayasaki et al. 2015). However, in the case of NGC 5548, this is an unlikely explanation if we were to interpret all its properties in 
the context of a TDE. For instance, its optical narrow emission lines imply the presence of a classical narrow-line region and therefore a much longer-lived AGN. Similar arguments hold for the possibility of causing a semi-periodic light curve from a TDE in a binary SMBH system (Liu et al. 2009). Another possibility is that we have a TDE in addition to the permanent accretion disk of a long-lived AGN, and the TDE contributes extra accretion during each pericenter passage. This would boost the accretion onto the $\mathrm{BH}$ in a periodic fashion, but material must be added to the accretion flow very close to the event horizon because the viscous time of accreting through the disk is very long. Perhaps such material is added to the central part, which periodically increases the X-ray emission from the disk corona; this in turn illuminates the central disk and boosts the optical light emitted from its surface. X-ray illumination is very important in NGC 5548 (Kaastra et al. 2014; Mehdipour et al. 2015, 2016), so such possibilities cannot be excluded. A detailed discussion of such a scenario is beyond the scope of this paper. We note, however, that TDEs are very rare events (Rees 1990; Magorrian \& Tremaine 1999 , one event per inactive galaxy every $10^{4}-10^{5}$ years) even though rates can be higher in AGN (Karas \& Šubr 2007) and in the presence of SMBBHs (e.g., Ivanov et al. 2005; Chen et al. 2011). In any case, the chances of seeing such an event in only one nearby galaxy analyzed are very small, and we therefore consider it very unlikely. Similarly, Landt et al. (2015) concluded that a TDE is a very unlikely explanation in NGC 5548.

\subsection{Binary $\mathrm{BHS}$}

There are several scenarios involving binary BH systems in AGNs. These can be divided into two broad groups: One is where only one of the BHs has an accretion disk and a BLR associated with it. The other is the case where both BHs are accreting through their own disks. For roughly equal BH mass (the only case considered here), the average separation of the two is of order $20 \mathrm{ld}$, and hence there is only one dusty toroidal structure around the two. In general, such systems are thought to be the end result of a galaxy merger, where the two BHs from the two galaxies are at the final stage of merging (see Milosavljević \& Merritt 2001; Merritt \& Milosavljević 2005). Earlier studies of NGC 5548 suggest some evidence for a merger 0.6-1.0 Gyr ago (more details in Tyson et al. 1998; Steenbrugge et al. 2005; Slavcheva-Mihova \& Mihov 2011). Some simulations performed to characterize the SMBBH systems show the formation of a circumbinary disk, inside of which the two BHs are accreting matter and forming miniaccretion disks (e.g., Bogdanović et al. 2008, 2009; Hayasaki et al. 2008; MacFadyen \& Milosavljević 2008; Cuadra et al. 2009; Smailagić \& Bon 2015). Further out, the circumbinary disk cools and may form a torus. Blending BLRs has also been investigated (e.g., Shen \& Loeb 2010; Popović 2012), or in case of a high-mass-ratio system, only one shifting BLR may be seen. A second $\mathrm{BH}$ can give rise to a host of phenomena that can yield periodic signals (see Katz 1997; Sillanpaa et al. 1988; Bogdanović et al. 2008; Bon et al. 2012; Kun et al. 2014; Graham et al. 2015a, 2015b). A detailed scenario of this type has been investigated in several papers by Bogdanović and collaborators (Bogdanović et al. 2008, 2009) who simulated a high-mass-ratio system with nearly identical time interval and periodicity as found here. These involve disk disruption, the formation and destruction of spiral arms in the gas between the BHs, and more. Some features of this model are appealing, especially those corresponding to periodic changes in the velocity curve of part of the gas, including cases where only one side of the profile is affected. Unfortunately, there have been no attempts to use the results of the dynamical simulation to calculated the resulting emission line spectrum, line profile, and time variations in the systems. While we are not in a position to look into this in detail, we note that the gas configuration in this model, and the gas properties, may be very different from what is known from many years of study of NGC 5548. Moreover, as noted earlier, the broadline spectrum of this source is very similar to the spectra of thousands of other type-I AGN. We thus consider it less plausible that NGC 5548 contains such a binary $\mathrm{BH}$ system.

A binary $\mathrm{BH}$ system where only one of the BHs carries its own disk and BLR (although the outskirts of the BLR must be disturbed by the "naked" $\mathrm{BH}$ ) is perhaps easier to explain. Obscuration in this scenario is very inefficient. However, we note that gravitational lensing of the luminous disk around the primary $\mathrm{BH}$ by the second $\mathrm{BH}$ can enhance the continuum emission by a factor of order 1.11 over a period of a few hundred days; the effects over BLR emission would be even smaller, by a factor of less then $1 \%$. For example, if both BHs have a mass of $5 \times 10^{7} M_{\odot}$ and their separation is $20 \mathrm{ld}$, the size of the Einstein ring is $\approx 0.48 \mathrm{ld}$. This should be compared with the size of the disk at $5100 \AA(\approx 2$ ld $)$. Such an enhancement is achromatic, which gives the immediate prediction that other continuum wavelengths would show an identical change of amplitude during the passage. Such a scenario cannot explain the periodic $\mathrm{H} \beta$ variations or its periodic light curve. Moreover, $15 \mathrm{ld}$ is well inside the BLR, so dynamical changes in the line-emitting gas must be considered too.

Using a radial velocity test for supermassive BBHs for broad, double-peaked emission lines (Liu et al. 2016) assuming equal mass components, the line peaks should be at about 2300 $\mathrm{km} \mathrm{s}^{-1}$. At some epochs, very small moving peaks in emission lines are identified corresponding to such velocities (see Shapovalova et al. 2004). However, it is very clear that the red and blue wings of $\mathrm{H} \beta$ respond to the same continuum variability at roughly the same time, so the gas emitting them is approximately at the same distance from the primary accretion disk. Recently, Li et al. (2016) proposed a BBH scenario in NGC 5548, which was a result of their two Gaussian decomposition model, fitted into 150 day averaged spectra. We tried to test these claims using a series of two Gaussian decomposition models with different types of constraints (e.g., a constant intensity ratio with a significant width difference of each Gaussian, forcing them to fit different parts of the line: narrower fits for the core and wider for the wings). We found that such a configuration could result in Gaussian components that switch sides and cross from blue to red and vice versa. Unfortunately, in every case tested (assuming constant initial parameters of component shifts), there were additional crossings in radial velocity curves that ruined the expected periodicity. We also note that in our modeling with a single Gaussian fit to the spectra (Figure 7), we obtained a radial velocity curve that appeared somewhat similar to the one of the $75 \%$ red half width (see Figures 7 and 3 ). This could imply the possibility of the same periodicity and could indicate the presence of a high-mass-ratio system. According to this analysis, we again find a BBH hypothesis to be unlikely, 
except for a in high-mass-ratio system, which we can neither disprove nor support.

\subsection{Obscuration by Gas and Dust inside and outside the BLR}

This category includes several possibilities for obscuration of the central disk and part of the BLR by a moving object at a distance corresponding to a 15.7 year period. We consider two different possibilities: an object inside the BLR moving around the primary $\mathrm{BH}$, and an object that is part of an outflowing wind moving around the polar axis of the disk. Both scenarios correspond to a situation where the length of obscuration is a small fraction of the period, perhaps a year or less. This situation is consistent with the new scheme presented in Section 4, which confirms the periodicity but does not show whether it is sinusoidal or corresponds to only one short event of dimming or enhancing the radiation of the central source. A large dust-free cloud moving around the primary $\mathrm{BH}$, inside the BLR, at a distance corresponding to a period of 15.7 years, can cause the periodic obscuration of the central continuum source. This explanation is appealing because the required distance, about $15 \mathrm{ld}$, is exactly in the middle of the range of the multiyear RM size of the $\mathrm{Hb}$ line, and coincidental agreement between the two distances is unlikely. However, there are various difficulties to this scenario. The obscuring material must be thick enough and large enough to occult a large fraction of the central accretion disk, which is 2 ld in radius (see e.g., Fausnaugh et al. 2016). This dimension is larger than the typical size of BLR clouds that are of order $0.1 \mathrm{ld}$ across, assuming that the density and column density are $\approx 10^{10} \mathrm{~cm}^{-3}$ and $\approx 10^{14} \mathrm{~cm}^{-2}$, respectively (e.g., Netzer 2013). A dust-free cloud must be Compton thick to block the $5100 \AA$ continuum because the ionized column of such gas is only of order $10^{22-23} \mathrm{~cm}^{-2}$. Thus both the radial and lateral dimensions of such a cloud are orders of magnitude larger than those considered typical of the BLR (a collection of clumps adding up to the required dimensions is just as difficult to explain). Obscuration by a dusty gas cloud is easier to explain because a very small column density, corresponding to $A_{V}<1$, is all that is required to absorb much of the radiation at $5100 \AA$. However, 15 ld is well within the dust sublimation radius for this source, and such grains will not survive in this environment. One can consider a very large dusty cloud in a spiraling elliptical orbit (e.g., Netzer \& Marziani 2010) where, in this case, part of the dust is not sublimated because it is shielded from the central source radiation during a big part of the orbit. We did not explore this possibility in detail, but consider it problematic because efficient shielding of the dust from the radiation at wavelengths longer than the Lyman or Balmer continuum edges is hard to explain. Explaining the observed periodic variations in $\mathrm{L}(\mathrm{H} \beta)$, and the period in its velocity curve, is even more challenging. Obscuration by dusty gas spiraling along the polar axis as part of a large scale disk wind is an alternative explanation. Disk winds have been considered for years as a general scenario to explain both the BLR and the dusty torus structure around the BH (see e.g., Elvis 2000; Elitzur \& Shlosman 2006; Elitzur 2008; Czerny \& Hryniewicz 2011; Netzer 2013, 2015; Arav et al. 2015; Elitzur \& Netzer 2016). This geometry, which is sketched in Figure 9 , allows a period of 15.7 years at a distance much larger than 15 ld from the $\mathrm{BH}$, because material is rotating around the polar axis of the system with a small opening angle of about $30^{\circ}$ (as suggested earlier for this object; see e.g., Rokaki et al. 1993;

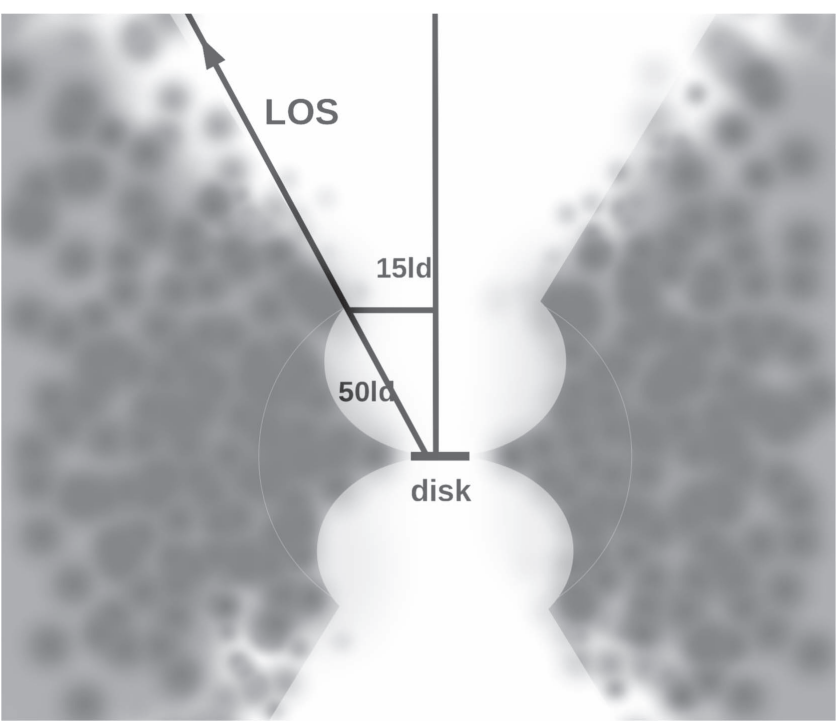

Figure 9. A representation of the disk obscuration. The partially obscured disk is seen through the inner edge of the torus cone (15 ld from the axes and at the distance from the disk of about $50 \mathrm{ld}$ ). Obscuration in such configurations is possible if the line of sight is similar to the cone opening angle. In this case it would correspond to angles of about $30^{\circ}$ or less. The obscuration model assumed here was inspired by various torus simulations obtained with the SKIRT code (Stalevski et al. 2016).

Kaastra et al. 2014) or less. For example, a dusty cloud at a distance of $50 \mathrm{ld}$, which is well inside the dust sublimation radius, can obscure the central source every 15.7 years for a few hundred days. The required column density is small, corresponding to $A_{V}<1 \mathrm{mag}$, and the lateral dimension is of order the disk size, $\sim 2$ ld (while we talk about "a cloud," this may well be a collection of clumps). Such a cloud can also obscure part of the BLR, although this fraction is much smaller because of the much larger dimensions of the BLR. Obscuration by dusty material must result in wavelength dependent reddening of the central continuum. In principle, the obscuration may last only a few hundred days every 15.7 years, and the available spectroscopy is not good enough to exclude such short-term reddening events. Another difficulty with spiraling out material is that the outward motion of the gas will result in a continuous change of radius and periodicity; this change may be small over the 43 years of observations considered here. These details are beyond the scope of the present work. The geometry of the torus considered here is very different from the simple tori considered in earlier studies (Netzer 2015, and references therein). This opens a range of possibilities that may be related to the periodicity discussed here, in particular the obscuration by line of sight dust. Large structures in the nonuniform torus wall, scattering by the torus dust, and so on should be investigated in detail.

\subsection{Periodic X-Ray Enhancement and Reflection}

The main difficulty in the previous scenarios is the lack of a clear connection between the periodic continuum variations, the periodic $\mathrm{H} \beta$ flux variations, and the periodic $\mathrm{H} \beta$ velocity variations. A more logical explanation is a mechanism that causes a periodic enhancement of the ionizing continuum, which in turn causes a periodic $\mathrm{H} \beta$ intensity variation (reverberation) that causes time-dependent changes in the mean emissivity radius of the $\mathrm{H} \beta$ line. In a virialized cloud 
system, an increase in the $\mathrm{H} \beta$ emission region is correlated to smaller gas velocities, which is in agreement with the observed line width variations. About $2 / 3$ of the bolometric luminosity in NGC 5548 is due to far-UV and soft X-ray emission (Gaskell 2008). The X-ray source is probably illuminating the central disk causing much of the optical-UV continuum variations (e.g., Uttley et al. 2003; Edelson et al. 2015; Fausnaugh et al. 2016). Accretion events close to the primary $\mathrm{BH}$, which enhance the emitted X-ray flux in a periodic fashion, can trigger the entire chain of events from optical continuum variations to $\mathrm{H} \beta$ flux variations to $\mathrm{H} \beta$ velocity variations. As suggested above, a pericenter passage of a partially tidally disrupted star is one possibility for such an event. An orbiting G2-like object (see Witzel et al. 2014) or some other stellar-size object with an orbit in a collision with the disk are other possibilities that could lead to such events. The energetics of these kinds of pericentric encounters depend on the consistency of the medium (the accretion disk or the torus) that is being crossed with their orbit. One can also imagine one or more gas clouds from the BLR in very eccentric orbit as the origin of such events. However, the mass of a single BLR cloud is tiny, which makes it very unstable against tidal forces, and its mass supply to the $\mathrm{BH}$ is many orders of magnitude below what is required to produce a significant $\mathrm{X}$-ray flare.

\subsection{Orbiting Body Crossing the Accretion Disk}

Periodic variations could be caused by an orbiting object perturbing the accretion disk while passing through it (e.g., Syer et al. 1991; Chakrabarti \& Wiita 1993; Armitage et al. 1996; Subr \& Karas 1999; Kieffer \& Bogdanović 2016; Nguyen \& Bogdanovic 2016; Pihajoki 2016). Such a scenario was proposed for NGC 5548 in Shapovalova et al. (2004) and was connected to the appearing and shifting bumpy features in the red wing of $\mathrm{H} \beta$. If we assume a stellar-mass object passing through the disk at a radius smaller than $15 \mathrm{ld}$, it could cause a perturbation in the disk and produce shocks (e.g., Chakrabarti \& Wiita 1993).

Such a collision could heat the disk (see e.g., Kieffer \& Bogdanovic 2016, raising the temperature above $10^{7} \mathrm{~K}$ ), producing periodic optical and X-ray emission. It is not our intention to attempt such calculations for NGC 5548, only to mention that the disk dimension and the orbit eccentricity are likely to be the limiting factors in such cases. The hot spots caused by impacts may not need to be present for the entire orbital time, but would be made periodically with each collision. Knowing that in our Galaxy there are a number of central stars that are on highly eccentric, randomly inclined orbits (i.e., the S0 stars; Eckart \& Genzel 1997; Ghez et al. 1998; Gillessen et al. 2009), and some show similar periodicities (e.g., for S0-2 the periodicity is 15.2 years and for S0-14 it is 38 years, Zucker et al. 2006; for S0-102 it is 11.5 years, see Meyer et al. 2012), it is conceivable that one could find objects on inclined orbits that could cross the accretion disk of NGC 5548. In the case of a star passing through the part of the disk responsible for the optical emission at about $2 \mathrm{ld}$ radius, the eccentricity of the orbit would be about 0.7 .

\section{CONCLUSIONS}

We analyzed the $5100 \AA$ continuum and the $\mathrm{H} \beta$ light and radial velocity curves of NGC 5548 using about 1600 spectra
Table 4

Slit Size and Position Angle Influence on Flux Calibration Using [O III] Emission

\begin{tabular}{lccc}
\hline \hline PA & Col. Range & $F_{\text {tot }}$ & $F_{\text {slit }}$ \\
\hline \multirow{4}{*}{$0-45$} & \multicolumn{2}{c}{2 arcsec Virtual Slit } \\
& $23-42$ & $4.28 \mathrm{E}-13$ & \\
& $23-42$ & $4.19 \mathrm{E}-13$ & $4.01 \mathrm{E}-13$ \\
$0-45$ & \multicolumn{2}{c}{1 arcsec Virtual Slit } \\
& $28-37$ & $4.03 \mathrm{E}-13$ & $4.12 \mathrm{E}-13$ \\
& $28-37$ & $3.90 \mathrm{E}-13$ & $3.86 \mathrm{E}-13$ \\
& & & $3.85 \mathrm{E}-13$ \\
\hline
\end{tabular}

spanning 43 years, including 12 years of new data. The main results of the study are as follows:

1. The continuum light curve shows a of periodicity of about 5700 days at a high confidence level. Similar periodicities are found in the light and radial velocity curves of the broad $\mathrm{H} \beta$ emission line. The period has been detected through a standard periodogram analysis, which we do not consider very significant, as well as through a new method specifically devised for the present data set that takes into account its heterogeneous quality and uneven sampling.

2. The detected periodicity is consistent with orbital motion inside the BLR of the source.

3. We examined various physical scenarios that can explain the observed periodicity. These include binary $\mathrm{BHs}$, the TDE of a massive star, orbiting dust-free and dusty clouds around the central $\mathrm{BH}$ and the polar axis of the system (in a polar wind), and periodic enhancement of the inner part of the disk producing the X-ray emission. While none of these can explain all the observations, the preferred explanation is the one linking the enhanced continuum, the enhanced line emission, and lowering the velocity through a single scenario related to the X-ray emission in this source. The enhanced X-ray emission could be triggered by an orbiting object periodically colliding with the accretion disk.

We would like to thank the International AGN Watch group for the spectra available on their website, especially Bradley Peterson for with kind permission to use unpublished spectra observed at Asiago. We would also like to thank Tamara Bogdanović, Jack Sulentic, and Mike Eraclous for all comments and suggestions. This research is part of projects 176003 "Gravitation and the large scale structure of the Universe" and 176001 "Astrophysical spectroscopy of extragalactic objects" supported by the Ministry of Education and Science of the Republic of Serbia. This work was supported by CONACyT research grant 151494 (Mexico), INTAS (grant N96-0328), and RFBR (grants N97-02-17625, N00-02-16272, N03-02-17123, 06-02-16843, N09-02-01136, 12-02-00857a, 12-02-01237a, N15-02-02101). MS acknowledges support by FONDECYT through grant No. 3140518.

\section{APPENDIX \\ [O III] CALIBRATION OF SLIT SPECTRA}

NGC 5548 hosts a very compact narrow-line region (NLR) structure (see Kraemer et al. 1998; Schmitt et al. 2003; Peterson et al. 2013). The [O III] emission is so tightly concentrated that there is no position angle (PA) effect if the slit is wide enough, as can be seen in Schmitt et al. (2003). For a $1^{\prime \prime}-2^{\prime \prime}$ slit, the 

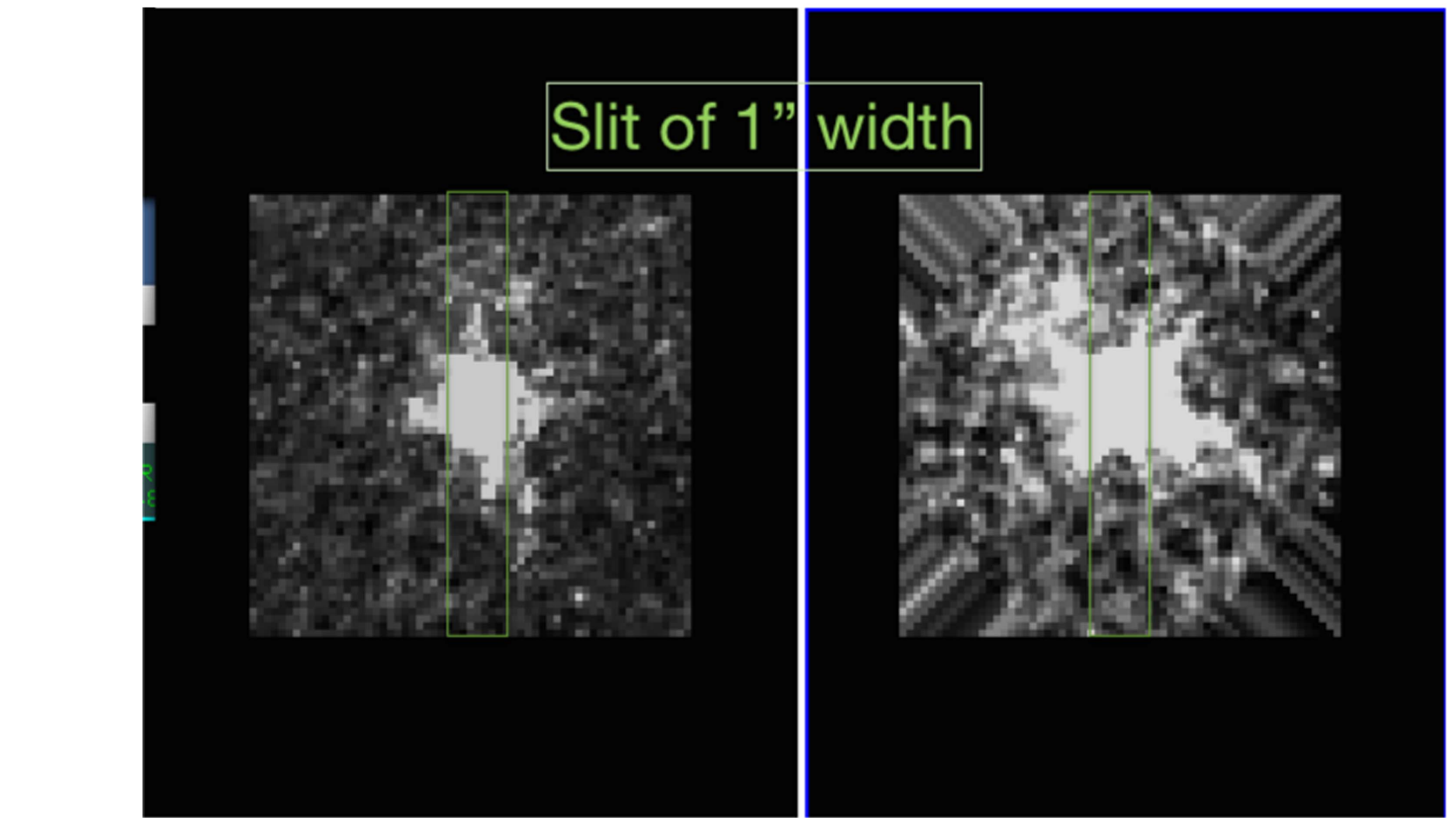

Figure 10. Slit size and position angle influence on flux calibration using [O III] emission (see Table 4). Left panel: PA $=0$. Right panel: same image rotated clockwise by $45^{\circ}$.

effect is small but appreciable. It is about $4 \%$ for a $1^{\prime \prime}$ slit, as can be seen by comparing the total flux within the slit at position angles $0^{\circ}$ and $-45^{\circ}$. This is shown in column 1 of Table 4, where the total [O III] flux within the slit $F_{\text {tot }}$ (column 3 ) is compared to the unresolved core flux within the slit $F_{\text {slit }}($ column 4$)$. The measurements were carried out on a narrow-band WFPC2 image with angular resolution 0 ". 1 (see Figure 10), which is available in digital format at $\mathrm{NED}^{19}$ and was originally published by Schmitt et al. (2003). The total emission measured on the image is $4.30 \times 10^{-13}$, assuming zero background in the image. The average background is actually slightly less than zero $\left(\approx-5 \times 10^{-17}\right)$, but it is difficult to estimate it accurately.

\section{REFERENCES}

Anderson, K. S. 1971, ApJ, 169, 449

Arav, N., Chamberlain, C., Kriss, G. A., et al. 2015, A\&A, 577, A37 Armitage, P. J., Zurek, W. H., \& Davies, M. B. 1996, ApJ, 470, 237 Begelman, M. C., Blandford, R. D., \& Rees, M. J. 1980, Natur, 287, 307 Bentz, M. C., Denney, K. D., Cackett, E. M., et al. 2007, ApJ, 662, 205 Bentz, M. C., Denney, K. D., Grier, C. J., et al. 2013, ApJ, 767, 149 Bentz, M. C., \& Katz, S. 2015, PASP, 127, 67

Bentz, M. C., Walsh, J. L., Barth, A. J., et al. 2009, ApJ, 705, 199

Bogdanović, T. 2015, ASSP, 40, 103

Bogdanović, T., Eracleous, M., \& Sigurdsson, S. 2009, NewAR, 53, 113

Bogdanović, T. S., Britton, D., Sigurdsson, S., \& Eracleous, M. 2008, ApJS, 174,455

Bon, E., Jovanović, P., Marziani, P., et al. 2012, ApJ, 759, 118

Bon, N., Popović, L. Č., \& Bon, E. 2014, AdSpR, 54, 1389

Bouchard, A., Prugniel, P., Koleva, M., \& Sharina, M. 2010, A\&A, 513, 54

Chakrabarti, S. K., \& Wiita, P. J. 1993, ApJ, 411, 602

Chakrabarti, S. K., \& Wiita, P. J. 1994, ApJ, 434, 518

Chen, X., Sesana, A., Madau, P., \& Liu, F. K. 2011, ApJ, 729, 13

Cherepashchuk, A. M., \& Lyutyi, V. M. 1973, ApL, 13, 165

\footnotetext{
$\overline{19}$ http://ned.ipac.caltech.edu/cgi-bin/ex_refcode?refcode=2003ApJS. $148 . .327 \mathrm{~S}$
}

Clavel, J., Reichert, G. A., Alloin, D., et al. 1991, ApJ, 366, 64 Cuadra, J., Armitage, P. J., Alexander, R. D., \& Begelman, M. C. 2009 MNRAS, 393, 1423

Czerny, B., \& Hryniewicz, K. 2011, A\&A, 525, L8

Czerny, B., Schwarzenberg-Czerny, A., \& Loska, Z. 1999, MNRAS, 303, 148 Czerny, B. 2006, in ASP Conf. Ser. 360, AGN Variability from X-Rays to Radio Waves, ed. C. M. Gaskell (San Francisco, CA: ASP), 265

De Paolis, F., Ingrosso, G., Nucita, A. A., \& Zakharov, A. F. 2003, A\&A, 410, 741

De Rosa, G., Peterson, B. M., Ely, J., et al. 2015, ApJ, 806, 128

Deutsch, A. N. 1966, IAUTB, 12, 252

Denney, K. D. 2010, ProQuest issertations And Theses, PhD thesis, The Ohio State Univ. Vol. 71-11, 101

Denney, K. D., De Rosa, G., Croxall, K., et al. 2014, ApJ, 796, 134

Denney, K. D., Peterson, B. M., Pogge, R. W., et al. 2009, ApJL, 704, L80

Dibai, É A., Esipov, V. F., \& Pronik, V. I. 1968, SvA, 11, 553

Dietrich, M., Bender, C. F., Bergmann, D. J., et al. 2001, A\&A, 371, 79

Dietrich, M., Kollatschny, W., Peterson, B. M., et al. 1993, ApJ, 408, 416

Du, P., Hu, C., Lu, K.-X., et al. 2015, ApJ, 806, 22

Eckart, A., \& Genzel, R. 1997, MNRAS, 284, 576

Edelson, R., Gelbord, J. M., Horne, K., et al. 2015, ApJ, 806, 129

Elitzur, M. 2008, NewAR, 52, 274

Elitzur, M., \& Netzer, H. 2016, MNRAS, 459, 585

Elitzur, M., \& Shlosman, I. 2006, ApJL, 648, L101

Elvis, M. 2000, ApJ, 545, 63

Eracleous, M., Boroson, T. A., Halpern, J. P., \& Liu, J. 2012, ApJS, 201, 23 Eracleous, M., \& Halpern, J. P. 2003, ApJ, 599, 886

Fan, J. H., Xie, G. Z., Pecontal, E., Pecontal, A., \& Copin, Y. 1998, ApJ, 507, 173

Fausnaugh, M. M., Denney, K. D., Barth, A. J., et al. 2016, ApJ, 821, 56

Flohic, H. M. L. G., \& Eracleous, M. 2008, ApJ, 686, 138

Gaskell, C. M. 1983, in Liege International Astrophysical Coll. 24, 473

Gaskell, C. M. 1988, ApJ, 325, 114

Gaskell, C. M. 2008, RMxAC, 32, 1

Gaskell, C. M. 2009, NewAR, 55, 140

Gaskell, C. M., \& Klimek, E. S. 2003, A\&AT, 22, 661

Gaskell, C. M., \& Sparke, L. S. 1986, ApJ, 305, 175

Gezari, S., Chornock, R., Rest, A., et al. 2012, Natur, 485, 217

Gezari, S., Halpern, J. P., \& Eracleous, M. 2007, ApJS, 169, 167

Ghez, A. M., Klein, B. L., Morris, M., \& Becklin, E. E. 1998, ApJ, 509, 678 Gillessen, S., Eisenhauer, F., Trippe, S., et al. 2009, ApJ, 692, 1075

Goosmann, R. W., Gaskell, C. M., \& Marin, F. 2014, AdSpR, 54, 1341 
Graham, M. J., Djorgovski, S. G., Stern, D., et al. 2015a, Natur, 518, 74 Graham, M. J., Djorgovski, S. G., Stern, D., et al. 2015b, MNRAS, 453, 1562 Guo, D., Tao, J., \& Qian, B. 2006, PASJ, 58, 503

Guo, D.-F., Hu, S.-M., Tao, J., et al. 2014, RAA, 14, 923

Hayasaki, K., Mineshige, S., \& Ho, L. C. 2008, ApJ, 682, 1134

Hayasaki, K., Stone, N. C., \& Loeb, A. 2015, arXiv:1501.05207

Hills, J. G. 1975, Natur, 254, 295

Hu, C., Wang, J.-M., Ho, L. C., et al. 2008, ApJL, 683, L115

Ivanov, P. B., Polnarev, A. G., \& Saha, P. 2005, MNRAS, 358, 1361

Jovanović, P., Popović, L. Č., Stalevski, M., \& Shapovalova, A. I. 2010, ApJ, 718,168

Kaastra, J. S., Kriss, G. A., Cappi, M., et al. 2014, Sci, 345, 64

Karas, V., \& Šubr, L. 2007, A\&A, 470, 11

Kaspi, S., Smith, P. S., Netzer, H., et al. 2000, ApJ, 533, 631

Katz, J. I. 1997, ApJ, 478, 527

Kieffer, T. F., \& Bogdanović, T. 2016, ApJ, 823, 155

Koleva, M., Bouchard, A., Prugniel, P., de Rijcke, S., \& Vauglin, I. 2013, MNRAS, 428, 2949

Koleva, M., Prugniel, P., Bouchard, A., \& Wu, Y. 2009, A\&A, 501, 1269

Koleva, M., Prugniel, P., de Rijcke, S., \& Zeilinger, W. W. 2011, MNRAS, 417, 1643

Koleva, M., Prugniel, P., Ocvirk, P., Le Borgne, D., \& Soubiran, C. 2008, MNRAS, 385, 1988

Kollatschny, W., \& Zetzl, M. 2013, A\&A, 551, L6

Komossa, S. 2006, MmSAI, 77, 733

Komossa, S. 2015, JHEAp, 7, 148

Komossa, S., \& Bade, N. 1999, A\&A, 343, 775

Komossa, S., Xu, D., \& Zensus, J. A. 2016, in IAU Symp. 312, Star Clusters and Galaxies across Cosmic Time (Cambridge: Cambridge Univ. Press), 63

Komossa, S., Xu, D., Zhou, H., Storchi-Bergmann, T., \& Binette, L. 2008, ApJ, 680, 926

Koratkar, A. P., \& Gaskell, C. M. 1991, ApJ, 375, 85

Korista, K. T., Alloin, D., Barr, P., et al. 1995, ApJS, 97, 285

Koshida, S., Minezaki, T., Yoshii, Y., et al. 2014, ApJ, 788, 159

Kovačević, J., Popović, L. Č., \& Dimitrijević, M. S. 2010, ApJS, 189, 15

Kraemer, S., Crenshaw, D. M., Fillipenko, A. V., Peterson, B. M., et al. 1998, ApJ, 499, 719

Kun, E., Gabányi, K. É, Karouzos, M., Britzen, S., \& Gergely, L. Á. 2014, MNRAS, 445, 1370

Landt, H., Ward, M. J., Steenbrugge, K. C., \& Ferland, G. J. 2015, MNRAS, 454, 3688

Lawson, C. L., \& Hanson, R. J. 1995, Solving Least Squares Problems (Philadelphia: SIAM)

Le Borgne, D., Rocca-Volmerange, B., Prugniel, P., et al. 2004, A\&A, 425,881

Lehto, H. J., \& Valtonen, M. J. 1996, ApJ, 460, 207

Lewis, K. T., Eracleous, M., \& Storchi-Bergmann, T. 2010, ApJS, 187, 416

Li, Y.-R., Wang, J.-M., Ho, L. C., et al. 2016, ApJ, 822, 4

Liu, F. K., Li, S., \& Chen, X. 2009, ApJL, 706, L133

Liu, J., Eracleous, M., \& Halpern, J. P. 2016, ApJ, 817, 42

Liu, T., Gezari, S., Heinis, S., et al. 2015, ApJL, 803, L16

Lomb, N. R. 1976, Ap\&SS, 39, 447

Lyutyi, V. M. 1973, ATsir, 777, 1

MacFadyen, A. I., \& Milosavljević, M. 2008, ApJ, 672, 83

Magorrian, J., \& Tremaine, S. 1999, MNRAS, 309, 447

Maoz, D., Smith, P. S., Jannuzi, B. T., Kaspi, S., \& Netzer, H. 1994, ApJ, 421, 34 Marquardt, D. W. 1963, SIAM, 11, 431

Marziani, P., Sulentic, J. W., Stirpe, G. M., et al. 2016, Ap\&SS, 361, 3

Mehdipour, M., Kaastra, J. S., Kriss, G. A., et al. 2015, A\&A, 575, A22

Mehdipour, M., Kaastra, J. S., Kriss, G. A., et al. 2016, A\&A, 588, A139

Merritt, D., \& Milosavljević, M. 2005, LRR, 8, 8

Meyer, L., Ghez, A. M., Schödel, R., et al. 2012, Sci, 338, 84

Milosavljević, M., \& Merritt, D. 2001, ApJ, 563, 34

Nandra, K., George, I. M., Mushotzky, R. F., Turner, T. J., \& Yaqoob, T. 1997, ApJ, 476, 70

Netzer, H. 2013, The Physics and Evolution of Active Galactic Nuclei (Cambridge: Cambridge Univ. Press)

Netzer, H. 2015, ARA\&A, 53, 365

Netzer, H., Maoz, D., Laor, A., et al. 1990, ApJ, 353, 108
Netzer, H., \& Marziani, P. 2010, ApJ, 724, 318

Netzer, H., \& Peterson, B. M. 1997, Astronomical Time Series, 218, 85

Nguyen, K., \& Bogdanovic, T. 2016, arXiv:1605.09389

Nikolajuk, M., Papadakis, I. E., \& Czerny, B. 2004, MNRAS, 350, L26

Oknyanskij, V., \& Lyuty, V. 2007, PZP, 7, 28

Oknyanskij, V. L. 1978, PZ, 21, 71

Peterson, B. M. 1997, An Introduction to Active Galactic Nuclei (Cambridge: Cambridge Univ. Press)

Peterson, B. M., Alloin, D., Axon, D., et al. 1992, ApJ, 392, 470

Peterson, B. M., Balonek, T. J., Barker, E. S., et al. 1991, ApJ, 368, 119

Peterson, B. M., Barth, A. J., Berlind, P., et al. 1999, ApJ, 510, 659

Peterson, B. M., Berlind, P., Bertram, R., et al. 2002, ApJ, 581, 197

Peterson, B. M., Denney, K. D., De Rosa, G., et al. 2013, ApJ, 779, 109

Peterson, B. M., \& Gaskell, C. M. 1986, AJ, 92, 552

Peterson, B. M., Korista, K. T., \& Cota, S. A. 1987, ApJ, 312, 1

Pihajoki, P. 2016, MNRAS, 457, 1145

Plavchan, P., Jura, M., Kirkpatrick, J. D., Cutri, R. M., \& Gallagher, S. C. 2008, ApJS, 175, 191

Popović, L. Č. 2012, NewAR, 56, 74

Popović, L. C., Shapovalova, A. I., Chavushyan, V. H., et al. 2008, PASJ, 60, 1

Pringle, J. E. 1996, MNRAS, 281, 357

Ptak, R. L., \& Stoner, R. E. 1973, ApJ, 179, 89

Rees, M. J. 1990, Sci, 247, 817

Rieger, F. M., \& Mannheim, K. 2000, A\&A, 359, 948

Rix, H.-W., \& White, S. D. M. 1992, MNRAS, 254, 389

Rokaki, E., Collin-Souffrin, S., \& Magnan, C. 1993, A\&A, 272, 8

Scargle, J. D. 1982, ApJ, 263, 835

Schmitt, H. R., Donley, J. L., Antonucci, R. R. J., Hutchings, J. B., \& Kinney, A. L. 2003, ApJS, 148, 327

Sergeev, S. G. 1992, Ap\&SS, 197, 77

Sergeev, S. G., Doroshenko, V. T., Dzyuba, S. A., et al. 2007, ApJ, 668, 708

Shapovalova, A. I., Burenkov, A. N., Borisov, N., et al. 2006, in ASP Conf. Ser. 360, AGN Variability from X-Rays to Radio Waves, ed. C. M. Gaskell (San Francisco, CA: ASP), 239

Shapovalova, A. I., Popović, L. Č., Chavushyan, V. H., et al. 2016, ApJS, 222,25

Shapovalova, A. I., Popović, L. Č., Collin, S., et al. 2008, A\&A, 486, 99

Shapovalova, A. I., Doroshenko, V. T., Bochkarev, N. G., et al. 2004, A\&A, 422, 925

Shen, Y., \& Loeb, A. 2010, ApJ, 725, 249

Sillanpaa, A., Haarala, S., Valtonen, M. J., Sundelius, B., \& Byrd, G. G. 1988, ApJ, 325, 628

Slavcheva-Mihova, L., \& Mihov, B. 2011, A\&A, 526, A43

Smailagić, M., \& Bon, E. 2015, JApA, 36, 513

Smith, H. J., \& Hoffleit, D. 1963, AJ, 68, 292

Stalevski, M., Ricci, C., Ueda, Y., et al. 2016, MNRAS, 458, 2288

Steenbrugge, K. C., Kaastra, J. S., Crenshaw, D. M., et al. 2005, A\&A, 434, 569

Stellingwerf, R. F. 1978, ApJ, 224, 953

Šubr, L., \& Karas, V. 1999, A\&A, 352, 452

Sudou, H., Iguchi, S., Murata, Y., \& Taniguchi, Y. 2003, Sci, 300, 1263

Syer, D., Clarke, C. J., \& Rees, M. J. 1991, MNRAS, 250, 505

Torres, G., Andersen, J., \& Giménez, A. 2010, A\&ARv, 18, 67

Tyson, J. A., Fischer, P., Guhathakurta, P., et al. 1998, AJ, 116, 102

Ulrich, M. H. 1972, ApJ, 174, 483

Uttley, P., Edelson, R., McHardy, I. M., Peterson, B. M., \& Markowitz, A. 2003, ApJL, 584, L53

Valtaoja, E., Teräsranta, H., Tornikoski, M., et al. 2000, ApJ, 531, 744

Valtonen, M., \& Ciprini, S. 2012, MmSAI, 83, 219

Valtonen, M., \& Sillanpää, A. 2011, AcPol, 51, 76

van der Marel, R. P. 1994, MNRAS, 270, 271

Vaughan, S., Uttley, P., Markowitz, A. G., et al. 2016, MNRAS, 461, 3145

Westman, D. B., MacLeod, C. L., \& Ivezić, Z. 2011, ASPC, 442, 159

Witzel, G., Ghez, A. M., Morris, M. R., et al. 2014, ApJL, 796, L8

Wu, Y., Singh, H. P., Prugniel, P., Gupta, R., \& Koleva, M. 2011, A\&A, 525,71

Zu, Y., Kochanek, C. S., \& Peterson, B. M. 2011, ApJ, 735, 80

Zucker, S., Alexander, T., Gillessen, S., Eisenhauer, F., \& Genzel, R. 2006, ApJL, 639, L21 